The Astrophysical Journal, 637:952-967, 2006 February 1

(C) 2006. The American Astronomical Society. All rights reserved. Printed in U.S.A.

\title{
SHEARING BOX SIMULATIONS OF THE MRI IN A COLLISIONLESS PLASMA
}

\author{
Prateek Sharma and Gregory W. Hammett \\ Princeton Plasma Physics Laboratory, Princeton University, Princeton, NJ 08543; psharma@pppl.gov, hammett@pppl.gov \\ ELIOT Quataert \\ Astronomy Department, University of California, Berkeley, CA 94720; eliot@astron.berkeley.edu \\ AND \\ JAMES M. STONE \\ Department of Astrophysical Sciences, Princeton University, Princeton, NJ 08544; jstone@astro.princeton.edu \\ Received 2005 August 23; accepted 2005 September 28
}

\begin{abstract}
We describe local shearing box simulations of turbulence driven by the magnetorotational instability (MRI) in a collisionless plasma. Collisionless effects may be important in radiatively inefficient accretion flows, such as near the black hole in the Galactic center. The MHD version of ZEUS is modified to evolve an anisotropic pressure tensor. A fluid closure approximation is used to calculate heat conduction along magnetic field lines. The anisotropic pressure tensor provides a qualitatively new mechanism for transporting angular momentum in accretion flows (in addition to the Maxwell and Reynolds stresses). We estimate limits on the pressure anisotropy due to pitch angle scattering by kinetic instabilities. Such instabilities provide an effective "collision" rate in a collisionless plasma and lead to more MHD-like dynamics. We find that the MRI leads to efficient growth of the magnetic field in a collisionless plasma, with saturation amplitudes comparable to those in MHD. In the saturated state, the anisotropic stress is comparable to the Maxwell stress, implying that the rate of angular momentum transport may be moderately enhanced in a collisionless plasma.
\end{abstract}

Subject headings: accretion, accretion disks — methods: numerical — MHD — plasmas — turbulence

\section{INTRODUCTION}

Following the seminal work of Balbus \& Hawley (1991), numerical simulations have demonstrated that magnetohydrodynamic (MHD) turbulence initiated by the magnetorotational instability (MRI) is an efficient mechanism for transporting angular momentum in accretion disks (e.g., Hawley et al. 1995, hereafter HGB95; see Balbus \& Hawley 1998 for a review). For a broad class of astrophysical accretion flows, however, the MHD assumption is not directly applicable. In particular, in radiatively inefficient accretion flow (RIAF) models for accretion onto compact objects, the accretion proceeds via a hot, low-density, collisionless plasma with the proton temperature larger than the electron temperature (see Narayan et al. 1998 and Quataert 2003 for reviews). In order to maintain such a two-temperature flow the plasma must be collisionless, and there are many cases in which the Coulomb mean free path is many orders of magnitude larger than the system size. Motivated by the application to RIAFs, this paper studies the nonlinear evolution of the MRI in a collisionless plasma, focusing on local simulations in the shearing box limit.

Quataert et al. (2000, hereafter QDH02) and Sharma et al. (2003, hereafter SHQ03) showed that the linear dynamics of the MRI in a collisionless plasma can be quite different from that in MHD. The maximum growth rate is a factor of $\sim 1.7$ larger and, perhaps more importantly, the fastest growing modes can shift to much longer wavelengths, giving direct amplification of longwavelength modes. Dynamical instability exists even when the magnetic tension forces are negligible because of the anisotropic pressure response in a collisionless plasma. In related work using Braginskii's anisotropic viscosity, Balbus (2004) has termed this modification of the MRI in the low-collisionality limit the "magnetoviscous" instability (see also Islam \& Balbus 2005).
In this paper, we are interested in simulating the dynamics of a collisionless plasma on length scales ( $\sim$ disk height) and timescales ( orbital period) that are very large compared to the microscopic plasma scales (such as the Larmor radius and the cyclotron period). Since the ratio of the size of the accretion flow to the proton Larmor radius is $\sim 10^{8}$ for typical RIAF models, direct particle methods such as PIC (particle in a cell), which need to resolve both of these scales, are computationally challenging and require simulating a reduced range of scales. Instead we use a fluid-based method to describe the large-scale dynamics of a collisionless plasma ("kinetic MHD," described in $\S 2$ ). The key differences with respect to MHD are that the pressure is a tensor rather than a scalar, anisotropic with respect to the direction of the local magnetic field, and that there are heat fluxes along magnetic field lines (related to Landau damping and wave-particle interactions). The drawback of our fluid-based method is, of course, that there is no exact expression for the heat fluxes if only a few fluid moments are retained in a weakly collisional plasma (the "closure problem"). We use results from Snyder et al. (1997, hereafter SHD97) who have derived approximations for the heat fluxes in terms of nonlocal parallel temperature and magnetic field gradients. These heat flux expressions can be shown to be equivalent to multipole Padé approximations to the $Z$-function involved in Landau damping. This approach can be shown to converge as more fluid moments of the distribution function are kept (Hammett et al. 1993), just as an Eulerian kinetic algorithm converges as more grid points in velocity space are kept. These fluid-based methods have been applied with reasonable success to modeling collisionless turbulence in fusion plasmas, generally coming within a factor of 2 of more complicated kinetic calculations in strong turbulence regimes (e.g., Dimits et al. 2000; Parker et al. 1994; Hammett et al. 1993; Scott 2005), although there can be larger differences in weak turbulence regimes (see Hammett 
et al. 1993; Dimits et al. 2000 and references therein). The simulations we report on here use an even simpler local approximation to the heat flux closures than those derived in SHD97. While not exact, these closure approximations allow one to begin to investigate kinetic effects with relatively fast modifications of fluid codes.

In a collisionless plasma the magnetic moment $\mu$ is an adiabatic invariant. Averaged over velocity space, this leads to conservation of $\langle\mu\rangle=p_{\perp} /(\rho B)$. As a result, pressure anisotropy with $p_{\perp}>p_{\|}$is created as the MRI amplifies the magnetic field in the accretion flow. This pressure anisotropy creates an anisotropic stress (like a viscosity!), which can be as important for angular momentum transport as the magnetic stress (as we show below). The pressure anisotropy cannot, however, grow without bound because high-frequency waves and kinetic microinstabilities feed on the free energy in the pressure anisotropy, effectively providing an enhanced rate of collisions that limit the pressure tensor anisotropy (leading to more MHD-like dynamics in a collisionless plasma). We capture this physics by using a subgrid model to restrict the allowed amplitude of the pressure anisotropy. This subgrid model (described in $\S 2.3$ ) is based on existing linear and nonlinear studies of instabilities driven by pressure anisotropy (e.g., Hasegawa 1969; Gary et al. 1997).

The remainder of this paper is organized as follows. In the next section $(\S 2)$ we describe Kulsrud's formulation of kinetic MHD (KMHD) and our closure model for the heat fluxes in a collisionless plasma. We also include a linear analysis of the MRI in the presence of a background pressure anisotropy and describe limits on the pressure anisotropy set by kinetic instabilities. In $\S 3$ we describe our modifications to the ZEUS code to model kinetic effects. Section 4 presents our primary results on the nonlinear evolution of the MRI in a collisionless plasma. In $\S 5$ we discuss these results, their astrophysical implications, and future work required to understand the global dynamics of collisionless accretion disks.

\section{GOVERNING EQUATIONS}

In the limit that all fluctuations of interest are at scales larger than the proton Larmor radius and have frequencies much smaller than the proton cyclotron frequency, a collisionless plasma can be described by the following magnetofluid equations (e.g., Kulsrud 1983; SHD97):

$$
\begin{gathered}
\frac{\partial \rho}{\partial t}+\nabla \cdot(\rho \boldsymbol{V})=0 \\
\rho \frac{\partial \boldsymbol{V}}{\partial t}+\rho(\boldsymbol{V} \cdot \nabla) \boldsymbol{V}=\frac{(\nabla \times \boldsymbol{B}) \times \boldsymbol{B}}{4 \pi}-\nabla \cdot \boldsymbol{P}+\boldsymbol{F}_{g}, \\
\frac{\partial \boldsymbol{B}}{\partial t}=\nabla \times(\boldsymbol{V} \times \boldsymbol{B}), \\
\boldsymbol{P}=p_{\perp} \boldsymbol{I}+\left(p_{\|}-p_{\perp}\right) \hat{\boldsymbol{b}} \hat{\boldsymbol{b}}=p_{\perp} \boldsymbol{I}+\boldsymbol{\Pi},
\end{gathered}
$$

where $\rho$ is the mass density, $\boldsymbol{V}$ is the fluid velocity, $\boldsymbol{B}$ is the magnetic field, $\boldsymbol{F}_{g}$ is the gravitational force, $\hat{\boldsymbol{b}}=\boldsymbol{B} /|\boldsymbol{B}|$ is a unit vector in the direction of the magnetic field, and $\boldsymbol{I}$ is the unit tensor. In equation (3) an ideal Ohm's law is used, neglecting resistivity. In equation (4), $\boldsymbol{P}$ is the pressure tensor with different perpendicular $\left(p_{\perp}\right)$ and parallel $\left(p_{\|}\right)$components with respect to the background magnetic field, and $\boldsymbol{\Pi}=\hat{\boldsymbol{b}} \hat{\boldsymbol{b}}\left(p_{\|}-p_{\perp}\right)$ is the anisotropic stress tensor. (Note that $\Pi$ is not traceless in the convention used here.) $\boldsymbol{P}$ should in general be a sum over all species but in the limit where ion dynamics dominate and electrons just provide a neutralizing background, the pressure can be interpreted as the ion pressure. This is the case for hot accretion flows in which $T_{p} \gg T_{e}$.

The exact pressures $p_{\|}$and $p_{\perp}$ can be rigorously determined by taking moments of the drift kinetic equation (Kulsrud 1983),

$$
\begin{gathered}
\frac{\partial f_{s}}{\partial t}+\left(v_{\|} \hat{\boldsymbol{b}}+\boldsymbol{v}_{\boldsymbol{E}}\right) \cdot \nabla f_{s} \\
+\left[-\hat{\boldsymbol{b}} \cdot \frac{D \boldsymbol{v}_{\boldsymbol{E}}}{D t}-\mu \hat{\boldsymbol{b}} \cdot \nabla \boldsymbol{B}+\frac{e_{s}}{m_{s}}\left(E_{\|}+\frac{F_{g \|}}{e_{s}}\right)\right] \frac{\partial f_{s}}{\partial v_{\|}}=C\left(f_{s}\right),
\end{gathered}
$$

which is the asymptotic expansion of the Vlasov equation for the distribution function $f_{s}\left(\boldsymbol{x}, \mu, v_{\|}, t\right)$ for species " $s$ " with mass $m_{s}$ and charge $e_{s}$ in the limit $\rho_{s} / L \ll 1, \omega / \Omega_{s} \ll 1$, where $\rho_{s}$ and $\Omega_{S}$ are the gyroradius and gyrofrequency, respectively. In equation (5), $\boldsymbol{v}_{\boldsymbol{E}}=c(\boldsymbol{E} \times \boldsymbol{B}) / B^{2}$ is the perpendicular drift velocity, $\mu=\left(\boldsymbol{v}_{\perp}-\boldsymbol{v}_{\boldsymbol{E}}\right)^{2} / 2 B$ is the magnetic moment (a conserved quantity in the absence of collisions), $F_{g \|}$ is the component of the gravitational force parallel to the direction of the magnetic field, and $D / D t=\partial / \partial t+\left(v_{\|} \hat{\boldsymbol{b}}+\boldsymbol{v}_{\boldsymbol{E}}\right) \cdot \nabla$ is the particle Lagrangian derivative in configuration space. The fluid velocity $\boldsymbol{V}=\boldsymbol{v}_{\boldsymbol{E}}+\hat{\boldsymbol{b}} u_{\|}$, so the $\boldsymbol{E} \times \boldsymbol{B}$ drift is determined by the perpendicular component of equation (2). Other drifts such as grad $B$, curvature, and gravity $\times \boldsymbol{B}$ drifts are higher order in the drift kinetic MHD ordering and do not appear in this equation. In equation $(5), C\left(f_{s}\right)$ is the collision operator to allow for generalization to collisional regimes. Collisions can also be used to mimic rapid pitch angle scattering due to high-frequency waves that break $\mu$ invariance. The parallel electric field is determined by $E_{\|}=\sum_{s}\left(e_{s} / m_{s}\right) \hat{\boldsymbol{b}} \cdot \nabla \cdot \boldsymbol{P}_{s} /$ $\sum_{S}\left(n_{s} e_{S}^{2} / m_{S}\right)$ (Kulsrud 1983), which ensures quasineutrality.

Separate equations of state for the parallel and perpendicular pressures can also be obtained from the moments of the drift kinetic equation (Chew et al. 1956). Neglecting the collision term these are

$$
\begin{aligned}
\rho B \frac{D}{D t}\left(\frac{p_{\perp}}{\rho B}\right) & =-\nabla \cdot \boldsymbol{q}_{\perp}-q_{\perp} \nabla \cdot \hat{\boldsymbol{b}}, \\
\frac{\rho^{3}}{B^{2}} \frac{D}{D t}\left(\frac{p_{\|} B^{2}}{\rho^{3}}\right) & =-\nabla \cdot \boldsymbol{q}_{\|}+2 q_{\perp} \nabla \cdot \hat{\boldsymbol{b}},
\end{aligned}
$$

where $D / D t=\partial / \partial t+\boldsymbol{V} \cdot \nabla$ is the fluid Lagrangian derivative and $\boldsymbol{q}_{\|, \perp}=q_{\|, \perp} \hat{\boldsymbol{b}}$ are the heat fluxes (flux of $p_{\|}$and $p_{\perp}$ ) parallel to the magnetic field. The equation for the magnetic moment density $\rho\langle\mu\rangle=p_{\perp} / B$ can be written in a conservative form:

$$
\frac{\partial}{\partial t}\left(\frac{p_{\perp}}{B}\right)+\nabla \cdot\left(\frac{p_{\perp}}{B} \boldsymbol{V}\right)=-\nabla \cdot\left(\frac{q_{\perp}}{B} \hat{b}\right) .
$$

If the heat fluxes are neglected (called the CGL or double adiabatic limit), as the magnetic field strength $(B)$ increases, $p_{\perp}$ increases $\left(p_{\perp} \propto \rho B\right)$, and $p_{\|}$decreases $\left(p_{\|} \propto \rho^{3} / B^{2}\right)$. Integrating equation (8) over a finite periodic (even a shearing periodic) box shows that $\left\langle p_{\perp} / B\right\rangle$ is conserved, where angle brackets denote a volume average. This implies that even when $q_{\|, \perp} \neq 0, p_{\perp}$ increases in a volume-averaged sense as the magnetic energy in the box increases. This means that for a collisionless plasma, pressure anisotropy $p_{\perp}>(<) p_{\|}$is created as a natural consequence of processes that amplify (reduce) $B$. This pressure anisotropy is crucial for understanding magnetic field amplification in collisionless dynamos.

To solve the set of equations (1)-(4), (6), and (7) in a simple fluid based formalism, we require expressions for $q_{\|}$and $q_{\perp}$ in 
terms of lower order moments. No simple exact expressions for $q_{\|}$and $q_{\perp}$ exist for nonlinear collisionless plasmas. Although simple, the double adiabatic or CGL approximation (where $q_{\|}=$ $q_{\perp}=0$ ) does not capture key kinetic effects such as Landau damping. In the moderately collisional limit $\left(\rho_{i}<\right.$ mean free path $<$ system size), where the distribution function is not very different from a local Maxwellian, one can use the Braginskii equations (Braginskii 1965) to describe anisotropic transport (see Balbus 2000, 2004, for astrophysical applications). However, in the hot RIAF regime, the mean free path is often much larger than the system size and the Braginskii equations are not formally applicable, although they are still useful as a qualitative indication of the importance of kinetic effects. The collisional limit of the kinetic MHD equations can be shown to recover the dominant anisotropic heat flux and viscosity tensor of Braginskii (SHD97). The local approximation to kinetic MHD that we use here leads to equations that are similar in form to Braginskii MHD, but with separate dynamical equations for parallel and perpendicular pressures. We also add models for enhanced pitch angle scattering by microinstabilities, which occur at very small scales and high frequencies beyond the range of validity of standard kinetic MHD. ${ }^{1}$

Hammett and collaborators have developed approximate fluid closures (called Landau fluid closure) for collisionless plasmas (Hammett \& Perkins 1990; Hammett et al. 1992; SHD97) that capture kinetic effects such as Landau damping. SHD97 give the resulting expressions for parallel heat fluxes $\left(q_{\|}, q_{\perp}\right)$ to be used in equations (6) and (7). Landau closures are based on Padé approximations to the full kinetic plasma dispersion function that reproduce the correct asymptotic behavior in both the $\omega / k_{\|} c_{\|} \gg 1$ and $\omega / k_{\|} c_{\|} \ll 1$ regimes (and provide a good approximation in between), where $\omega$ is the angular frequency, $k_{\|}$is the wavenumber parallel to the magnetic field, and $c_{\|}=\left(p_{\|} / \rho\right)^{1 / 2}$ is the parallel thermal velocity of the particles. In Fourier space, the linearized heat fluxes can be written as (eqs. [39] and [40] in SHD97)

$q_{\|}=-\sqrt{\frac{8}{\pi}} \rho_{0} c_{\| 0} \frac{i k_{\|}\left(p_{\|} / \rho\right)}{\left|k_{\|}\right|}$,

$q_{\perp}=-\sqrt{\frac{2}{\pi}} \rho_{0} c_{\| 0} \frac{i k_{\|}\left(p_{\perp} / \rho\right)}{\left|k_{\|}\right|}+\sqrt{\frac{2}{\pi}} c_{\| 0} \frac{p_{\perp 0}}{B_{0}}\left(1-\frac{p_{\perp 0}}{p_{\| 0}}\right) \frac{i k_{\|} B}{\left|k_{\|}\right|}$,

where 0 subscripts indicate equilibrium quantities. Real space expressions are somewhat more cumbersome and are given by convolution integrals

$$
\begin{aligned}
q_{\|}= & -\left(\frac{2}{\pi}\right)^{3 / 2} n_{0} c_{\| 0} \int_{0}^{\infty} \delta z^{\prime} \frac{T_{\|}\left(z+z^{\prime}\right)-T_{\|}\left(z-z^{\prime}\right)}{z^{\prime}}, \\
q_{\perp}= & -\left(\frac{2}{\pi^{3}}\right)^{1 / 2} n_{0} c_{\| 0} \int_{0}^{\infty} \delta z^{\prime} \frac{T_{\perp}\left(z+z^{\prime}\right)-T_{\perp}\left(z-z^{\prime}\right)}{z^{\prime}} \\
& +\left(\frac{2}{\pi^{3}}\right)^{1 / 2} c_{\| 0}\left(1-\frac{p_{\perp 0}}{p_{\| 0}}\right) \frac{p_{\perp 0}}{B_{0}} \int_{0}^{\infty} \delta z^{\prime} \frac{B\left(z+z^{\prime}\right)-B\left(z-z^{\prime}\right)}{z^{\prime}},
\end{aligned}
$$

where $n_{0}$ is the number density, $T_{\|}=p_{\|} / n$ and $T_{\perp}=p_{\perp} / n$ are the parallel and perpendicular temperatures, and $z^{\prime}$ is the spatial vari-

\footnotetext{
1 This would also be needed when using Braginskii equations, because they are not necessarily well posed in situations where the anisotropic stress tensor can drive arbitrarily small-scale instabilities (Schekochihin et al. 2005).
}

able along the magnetic field line. SHQ03 have shown that these fluid closures for the heat fluxes accurately reproduce the kinetic linear Landau damping rate for all MHD modes (slow, Alfvén, fast and entropy modes). The growth rate of the MRI using the Landau closure model is also very similar to that obtained from full kinetic theory. As noted in the introduction, in addition to reproducing linear modes/instabilities, Landau fluid closures have also been used to model turbulence in fusion plasmas with reasonable success.

These closure approximations were originally developed for turbulence problems in fusion energy devices with a strong guide magnetic field, where the parallel dynamics is essentially linear and fast Fourier transforms (FFTs) could be easily used to quickly evaluate the Fourier expressions above. In astrophysical problems with larger amplitude fluctuations and tangled magnetic fields, evaluation of the heat fluxes become somewhat more complicated. One could evaluate the convolution expressions, equations (11) and (12) (with some modest complexity involved in writing a subroutine to integrate along magnetic field lines), leading to a code with a computational time $T_{\text {cpu }} \propto N_{x}^{3} N_{\|}$, where $N_{x}^{3}$ is the number of spatial grid points and $N_{\|}$is the number of points kept in the integrals along field lines. (In some cases, it may be feasible to map the fluid quantities to and from a field line following coordinate system so that FFTs can reduce this to $T_{\text {cpu }} \propto N_{x}^{3} \log N_{\|}$.) While this is more expensive than simple MHD where $T_{\text {cpu }} \propto N_{x}^{3}$, it could still represent a savings over a direct solution of the drift kinetic equation, which would require $T_{\text {cpu }} \propto N_{x}^{3} N_{v_{\|}} N_{v_{\perp}}$, where $N_{v_{\|}} N_{v_{\perp}}$ is the number of grid points for velocity space. ${ }^{2}$

As a first step for studying kinetic effects, in this paper we pick out a characteristic wavenumber $k_{\mathrm{L}}$ that represents the scale of collisionless damping and use a local approximation for the heat fluxes in Fourier space, with a straightforward assumption about the nonlinear generalization:

$$
\begin{aligned}
& q_{\|}=-\sqrt{\frac{8}{\pi}} \rho c_{\|} \frac{\nabla_{\|}\left(p_{\|} / \rho\right)}{k_{\mathrm{L}}}, \\
& q_{\perp}=-\sqrt{\frac{2}{\pi}} \rho c_{\|} \frac{\nabla_{\|}\left(p_{\perp} / \rho\right)}{k_{\mathrm{L}}}+\sqrt{\frac{2}{\pi}} c_{\|} p_{\perp}\left(1-\frac{p_{\perp}}{p_{\|}}\right) \frac{\nabla_{\|} B}{k_{\mathrm{L}} B} .
\end{aligned}
$$

Note that this formulation of the heat flux is analogous to a Braginskii heat conduction along magnetic field lines. For linear modes with $\left|k_{\|}\right| \sim k_{\mathrm{L}}$, these approximations will of course agree with kinetic theory as well as the Padé approximations shown in (SHD97). One can think of $k_{\mathrm{L}}$ as approximately controlling the heat conduction rate, although this does not necessarily affect the resulting Landau damping rate of a mode in a monotonic way, since this sometimes exhibits impedance matching behavior. That is, some modes are weakly damped in both the small and large (isothermal) heat conduction limits. We vary $k_{\mathrm{L}}$ to investigate the sensitivity of our results to this parameter.

\subsection{Linear Modes}

Since pressure anisotropy arises as a consequence of magnetic field amplification in a collisionless plasma, it is of interest to repeat the linear analysis of the collisionless MRI done previously

\footnotetext{
2 On the other hand, an effective hyperdiffusion operator in velocity space may reduce the velocity resolution requirements, and recent direct kinetic simulations of turbulence in fusion devices have found that often one does not need very high velocity resolution. This may make a direct solution of the drift kinetic equation tractable for some astrophysical kinetic MHD problems. Furthermore, a direct solution of the drift kinetic equation involves only local operations and thus is somewhat easier to parallelize than the convolution integrals.
} 
in QDH02, but with a background pressure anisotropy $\left(p_{\| 0} \neq\right.$ $\left.p_{\perp 0}\right)$. We consider the simple case of a vertical magnetic field. This analysis provides a useful guide to understanding some of our numerical results.

We linearize equations (1)-(4) for a differentially rotating disk $\left[\boldsymbol{V}_{0}=R \Omega(R) \hat{\boldsymbol{\phi}}\right]$ with an anisotropic pressure about a uniform subthermal vertical magnetic field $\left(\boldsymbol{B}_{0}=B_{z} \hat{z}\right)$. We assume that the background (unperturbed) plasma is described by a bi-Maxwellian distribution $\left(p_{\| 0} \neq p_{\perp 0}\right)$. We also assume that the perturbations are axisymmetric, of the form $\exp [-i \omega t+i \boldsymbol{k} \cdot \boldsymbol{x}]$ with $\boldsymbol{k}=k_{R} \hat{\boldsymbol{R}}+$ $k_{z} \hat{z}$. Writing $\rho=\rho_{0}+\delta \rho, \boldsymbol{B}=\boldsymbol{B}_{0}+\delta \boldsymbol{B}, p_{\perp}=p_{\perp 0}+\delta p_{\perp}, p_{\|}=$ $p_{\| 0}+\delta p_{\|}$, working in cylindrical coordinates, and making a $|k| R \gg 1$ assumption, the linearized versions of equations (1)(3) become

$$
\begin{aligned}
\omega \delta \rho= & \rho_{0} \boldsymbol{k} \cdot \delta \boldsymbol{v} \\
-i \omega \rho_{0} \delta v_{R} & -\rho_{0} 2 \Omega \delta v_{\phi}=-\frac{i k_{R}}{4 \pi} B_{z} \delta B_{z} \\
& +i k_{z}\left[\frac{B_{z}}{4 \pi}-\frac{\left(p_{\| 0}-p_{\perp 0}\right)}{B_{z}}\right] \delta B_{R}-i k_{R} \delta p_{\perp}, \\
-i \omega \rho_{0} \delta v_{\phi} & +\rho_{0} \delta v_{R} \frac{\kappa^{2}}{2 \Omega}=i k_{z}\left[\frac{B_{z}}{4 \pi}-\frac{\left(p_{\| 0}-p_{\perp 0}\right)}{B_{z}}\right] \delta B_{\phi}, \\
-i \omega \rho_{0} \delta v_{z}= & -i k_{R}\left(p_{\| 0}-p_{\perp 0}\right) \frac{\delta B_{R}}{B_{z}}-i k_{z} \delta p_{\|}, \\
\omega \delta B_{R}= & -k_{z} B_{z} \delta v_{R}, \\
\omega \delta B_{\phi}= & -k_{z} B_{z} \delta v_{\phi}-\frac{i k_{z} B_{z}}{\omega} \frac{d \Omega}{d \ln R} \delta v_{R}, \\
\omega \delta B_{z}= & k_{R} B_{z} \delta v_{R},
\end{aligned}
$$

where $\kappa^{2}=4 \Omega^{2}+d \Omega^{2} / d \ln R$ is the epicyclic frequency. Equations (15)-(21) describe the linear modes of a collisionless disk with an initial pressure anisotropy about a vertical magnetic field. This corresponds to the $\theta=\pi / 2$ case of QDH02, but with an anisotropic initial pressure. Equations (16) and (17) show that an initial anisotropic pressure modifies the Alfvén wave characteristics, so we expect a background pressure anisotropy to have an important effect on the MRI. One way of interpreting equations (16) and (17) is that $p_{\perp}>p_{\|}\left(p_{\|}>p_{\perp}\right)$ makes the magnetic fields more (less) stiff; as a result, this will shift the fastest growing MRI mode to larger (smaller) scales.

The linearized equations for the parallel and perpendicular pressure response are given by equations (33) and (34) in SHQ03. We present them here for the sake of completeness:

$$
\begin{aligned}
& -i \omega \delta p_{\|}+p_{\| 0} i \boldsymbol{k} \cdot \delta \boldsymbol{v}+i k_{z} q_{\|}+2 p_{\| 0} i k_{z} \delta v_{z}=0, \\
& -i \omega \delta p_{\perp}+2 p_{\perp 0} i \boldsymbol{k} \cdot \delta \boldsymbol{v}+i k_{z} q_{\perp}-p_{\perp 0} i k_{z} \delta v_{z}=0,
\end{aligned}
$$

where the heat fluxes can be expressed in terms of lower moments using

$$
\begin{aligned}
& q_{\perp}=-\sqrt{\frac{2}{\pi}} c_{\| 0} \frac{i k_{z}}{\left|k_{z}\right|}\left(\delta p_{\perp}-c_{\| 0}^{2} \delta \rho\right) \\
& +\sqrt{\frac{2}{\pi}} c_{\| 0} p_{\perp 0}\left(1-\frac{p_{\perp 0}}{p_{\| 0}}\right) \frac{i k_{z}}{\left|k_{z}\right|} \frac{\delta B}{B_{z}}, \\
& q_{\|}=-\sqrt{\frac{8}{\pi}} c_{\| 0} \frac{i k_{z}}{\left|k_{z}\right|}\left(\delta p_{\|}-c_{\| 0}^{2} \delta \rho\right),
\end{aligned}
$$

where $c_{\| 0}=\left(p_{\| 0} / \rho_{0}\right)^{1 / 2}$ and $\delta B=|\delta \boldsymbol{B}|$.

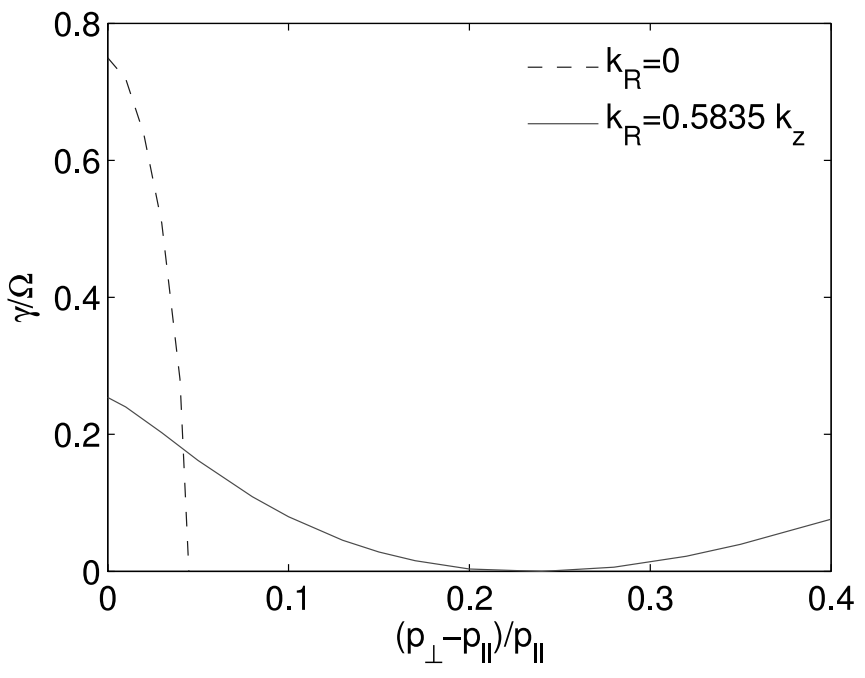

FIG. 1.-Normalized growth rate $(\gamma / \Omega)$ of the MRI vs. normalized pressure anisotropy, $\left(p_{\perp}-p_{\perp}\right) / p_{\|}$for $\beta=100, k_{z} V_{\mathrm{A} z} / \Omega=(15 / 16)^{1 / 2}$, and two different values of $k_{R}$. Note that even a small anisotropy can stabilize the fastest growing MRI mode. The growth at large pressure anisotropy for $k_{R} \neq 0$ is due to the mirror mode.

Figure 1 shows the MRI growth rate as a function of pressure anisotropy for two values of $k_{R}$ for $\beta=100$. This figure shows that the fastest growing MHD mode $\left(k_{R}=0\right)$ is stabilized for $\left(p_{\perp 0}-p_{\| 0}\right) / p_{\| 0} \sim 4 / \beta$; modes with $k_{R} \neq 0$ modes require larger anisotropy for stabilization. For $\beta \gg 1$, these results highlight that only a very small pressure anisotropy is required to stabilize the fastest growing MRI modes. Growth at large pressure anisotropies in Figure 1 for $k_{R} \neq 0$ mode is because of the mirror instability that is discussed below. The physical interpretation of the stabilization of the MRI in Figure 1 is that as the pressure anisotropy increases $\left(p_{\perp 0}>p_{\| 0}\right)$, the field lines effectively become stiffer and modes of a given $k$ can be stabilized (although longer wavelength modes will still be unstable).

In a numerical simulation in which the pressure anisotropy is allowed (unphysically, as we see in $\S 2.2$ ) to grow without bound as the magnetic field grows, this effect is capable of stabilizing all of the MRI modes in the computational domain at very small amplitudes (see Fig. 6, discussed in $\S 4$ ).

\subsection{Isotropization of the Pressure Tensor in Collisionless Plasmas}

Pressure anisotropy $\left(p_{\perp} \neq p_{\|}\right)$is a source of free energy that can drive instabilities that act to isotropize the pressure, effectively providing an enhanced "collision" rate in a collisionless plasma (e.g., Gary et al. 1997). In order to do so, the instabilities must break magnetic moment conservation and thus must have frequencies comparable to the cyclotron frequency and/or parallel wavelengths comparable to the Larmor radius. Because of the large disparity in timescales between $\mu$-breaking microinstabilities and the MRI $\left(\omega_{\text {micro }} / \Omega \sim 10^{8}\right)$, one can envision the microinstabilities as providing a "hard wall" limit on the pressure anisotropy: once the pressure anisotropy exceeds the threshold value where microinstabilities are driven and cause rapid pitch angle scattering, the pressure anisotropy nearly instantaneously reduces the anisotropy to its threshold value (from the point of view of the global disk dynamics). In this section we review the relevant instabilities that limit the pressure anisotropy in high- $\beta$ collisionless plasmasthese are the firehose, mirror, and ion cyclotron instabilities. We then discuss how we have implemented these estimated upper bounds on the pressure anisotropy in our numerical simulations. 


\subsubsection{Maximum Anisotropy for $p_{\|}>p_{\perp}$}

Plasmas with $p_{\|}>p_{\perp}$ can be unstable to the firehose instability, whose dispersion relation for parallel propagation is given by equation (2.12) of Kennel \& Sagdeev (1967):

$$
\omega^{2}-\omega \Omega_{i} k_{\|}^{2} \rho_{i}^{2}+\Omega_{i}^{2} k_{\|}^{2} \rho_{i}^{2}\left(1-\frac{p_{\perp}}{p_{\|}}-\frac{2}{\beta_{\|}}\right)=0,
$$

where $\beta_{\|}=8 \pi p_{\|} / B^{2}, \rho_{i}$ is the ion Larmor radius, $\Omega_{i}$ is the ion cyclotron frequency, and $k_{\|}$is the wavenumber parallel to the local magnetic field direction. Solving for $\omega$ gives

$$
\omega=k_{\|}^{2} \rho_{i}^{2} \frac{\Omega_{i}}{2} \pm i k_{\|} c_{\| 0}\left(1-\frac{p_{\perp}}{p_{\|}}-\frac{2}{\beta_{\|}}-\frac{k_{\|}^{2} \rho_{i}^{2}}{4}\right)^{1 / 2}
$$

For long wavelengths, the firehose instability requires $p_{\|}>p_{\perp}+$ $B^{2} / 4 \pi$ and is essentially an Alfvén wave destabilized by the pressure anisotropy. The maximum growth rate occurs when $k_{\|}^{2} \rho_{i}^{2}=$ $2\left(1-p_{\perp} / p_{\|}-2 / \beta_{\|}\right)$and is given by $\Omega_{i}\left(1-p_{\perp} / p_{\|}-2 / \beta_{\|}\right)$. We use an upper limit on $p_{\|}>p_{\perp}$ corresponding to $1-p_{\perp} / p_{\|}-$ $2 / \beta_{\|}<1 / 2$, which is an approximate condition for the growth of modes that will violate $\mu$ conservation and produce rapid pitch angle scattering.

\subsubsection{Maximum Anisotropy for $p_{\perp}>p_{\|}$}

For $p_{\perp}>p_{\|}$there are two instabilities that act to isotropize the pressure, the mirror instability and the ion cyclotron instability (e.g., Gary et al. 1997). A plasma is unstable to the mirror instability when $p_{\perp} / p_{\|}-1>1 / \beta_{\perp}$, although as discussed below, only for somewhat larger anisotropies is magnetic moment conservation violated. Formally, a plasma with any nonzero pressure anisotropy can be unstable to the ion cyclotron instability (Stix 1992). However, there is an effective threshold given by the requirement that the unstable modes grow on a timescale comparable to the disk rotation period.

Equations (43') and (44') of Hasegawa (1969) give the wavenumber for the fastest growing mirror mode,

$$
\begin{aligned}
& \frac{k_{\|}}{k_{\perp}}=\sqrt{\frac{(D-1)}{4},} \\
& k_{\perp} \rho_{i}=\sqrt{\frac{(D-1)}{6}},
\end{aligned}
$$

where $D=\beta_{\perp}\left(p_{\perp} / p_{\|}-1\right)$ and $\beta_{\perp}=8 \pi p_{\perp} / B^{2}$. To estimate the pressure anisotropy at which $\mu$ conservation is broken and thus pitch angle scattering is efficient, we calculate $D$ for which $k_{\|} \rho_{i} \sim$ $k_{\perp} \rho_{i} \sim 1$. This implies $D \approx 7$ or that $\mu$ conservation fails (and pitch angle scattering occurs) if the pressure anisotropy satisfies

$$
\frac{p_{\perp}}{p_{\|}}-1>\frac{7}{\beta_{\perp}} .
$$

The ion cyclotron instability can be also be excited when $p_{\perp}>$ $p_{\|}$. Gary and collaborators have analyzed the ion cyclotron instability in detail through linear analysis and numerical simulations. Gary et al. (1997) and Gary \& Lee (1994) calculate the pressure anisotropy required for a given growth rate $\gamma$ relative to the ion cyclotron frequency $\Omega_{i}$

$$
\frac{p_{\perp}}{p_{\|}}-1>\frac{S^{\prime}}{\beta_{\|}^{p}}
$$

where $S^{\prime}=0.35$ and $p=0.42$ are fitting parameters quoted in equation (2) of Gary \& Lee (1994) for $\gamma / \Omega_{i}=10^{-4}$. Moreover, for $\gamma \ll \Omega_{i}$ the threshold anisotropy depends only very weakly on the growth rate $\gamma$. As a result, equation (31) provides a reasonable estimate of the pressure anisotropy required for pitch angle scattering by the ion cyclotron instability to be important on a timescale comparable to the disk rotation period.

\subsection{Pressure Anisotropy Limits}

Motivated by the above considerations, we require that the pressure anisotropy satisfy the following inequalities in our simulations (at each grid point and for all time steps):

$$
\begin{aligned}
& \frac{p_{\perp}}{p_{\|}}-1+\frac{2}{\beta_{\|}}>\frac{1}{2}, \\
& \frac{p_{\perp}}{p_{\|}}-1<\frac{2 \xi}{\beta_{\perp}}, \\
& \frac{p_{\perp}}{p_{\|}}-1<S\left(\frac{2}{\beta_{\|}}\right)^{1 / 2},
\end{aligned}
$$

where $S$ and $\xi$ are constants described below. It is important to note that the fluid-based kinetic theory utilized in this paper can correctly reproduce the existence and growth rates of the firehose and mirror instabilities (although not the ion cyclotron instability). ${ }^{3}$ However, it can only do so for long-wavelength perturbations that conserve $\mu$. The relevant modes for pitch angle scattering occur at the Larmor radius scale, which is very small in typical accretion flows and is unresolved in our simulations. For this reason we must impose limits on the pressure anisotropy and cannot simultaneously simulate the MRI and the relevant instabilities that limit the pressure anisotropy. The algorithm to impose the pressure anisotropy limits is explained in Appendix A3.

In equation (33), the parameter $\xi$ determines the threshold anisotropy above which the mirror instability leads to pitch angle scattering. A value of $\xi=3.5$ was estimated in $\S 2.2 .2$. We take this as our fiducial value, but for comparison also describe calculations with $\xi=0.5$, which corresponds to the marginal state for the mirror instability. We compare both models because the saturation of the mirror instability is not well understood, particularly under the conditions appropriate to a turbulent accretion disk. Equation (34) is based on the pitch angle scattering model used by Birn \& Hesse (2001) for simulations of magnetic reconnection in collisionless plasmas; following them we choose $S=0.3$. Equation (34) with $S=0.3$ gives results that are nearly identical (for the typical range of $\beta$ studied here) to the pressure anisotropy threshold for the ion cyclotron instability discussed in $\S 2.2 .2$ (eq. [31]).

In our simulations we find that for typical calculations, if $\xi=0.5$, then equation (33) (the "mirror instability") dominates the isotropization of the pressure tensor, while if $\xi=3.5$, then equation (34) (the "ion cyclotron instability") dominates. We also find that our results are insensitive to the form of the $p_{\|}>p_{\perp}$ threshold (eq. [32]); e.g., simulations with $1-p_{\perp} / p_{\|}<2 / \beta_{\|}$(the marginal state of the firehose mode) instead of equation (32) give nearly identical results. Future fully kinetic simulations of the

\footnotetext{
3 The double adiabatic limit $\left(q_{\perp}=q_{\|}=0\right)$ predicts an incorrect threshold and incorrect growth rates for the mirror instability (e.g., SHD97). Thus, it is important to use the heat flux models described in $\S 2$ to capture the physics of the mirror instability.
} 
mirror, firehose, and ion cyclotron instabilities will be useful for calibrating the pitch angle scattering models used here.

\section{NUMERICAL METHODS}

In this section we discuss the shearing box equations that we solve numerically and the modifications made to ZEUS to include kinetic effects.

\subsection{Shearing Box}

The shearing box is based on a local expansion of the tidal forces in a reference frame corotating with the disk (see HGB95 for details). A fiducial radius $R_{0}$ in the disk is picked out and the analysis is restricted to a local Cartesian patch such that $L_{x}, L_{y}$, $L_{z} \ll R_{0}$ (where $x=r-R_{0}, y=\phi$ and $z=z$ ). In this paper only the radial component of gravity is considered and buoyancy effects are ignored. We also assume a Keplerian rotation profile. With these approximations, the equations of Landau MHD in the shearing box are

$$
\begin{gathered}
\frac{\partial \rho}{\partial t}+\nabla \cdot(\rho \boldsymbol{V})=0 \\
\frac{\partial \boldsymbol{V}}{\partial t}+\boldsymbol{V} \cdot \nabla \boldsymbol{V}=-\frac{1}{\rho} \nabla\left(p_{\perp}+\frac{B^{2}}{8 \pi}\right)+\frac{\boldsymbol{B} \cdot \nabla \boldsymbol{B}}{4 \pi \rho} \\
-\frac{1}{\rho} \nabla \cdot \boldsymbol{\Pi}-2 \Omega \times \boldsymbol{V}+3 \Omega^{2} x \hat{\boldsymbol{x}} \\
\frac{\partial \boldsymbol{B}}{\partial t}=\nabla \times(\boldsymbol{V} \times \boldsymbol{B}), \\
\frac{\partial p_{\|}}{\partial t}+\nabla \cdot\left(p_{\|} \boldsymbol{V}\right)+\nabla \cdot \boldsymbol{q}_{\|}+2 p_{\|} \hat{\boldsymbol{b}} \cdot \nabla \boldsymbol{V} \cdot \hat{\boldsymbol{b}}-2 q_{\perp} \nabla \cdot \hat{\boldsymbol{b}} \\
=-\frac{2}{3} \nu_{\mathrm{eff}}\left(p_{\|}-p_{\perp}\right), \\
\frac{\partial p_{\perp}}{\partial t}+\nabla \cdot\left(p_{\perp} \boldsymbol{V}\right)+\nabla \cdot \boldsymbol{q}_{\perp}+p_{\perp} \nabla \cdot \boldsymbol{V}-p_{\perp} \hat{\boldsymbol{b}} \cdot \nabla \boldsymbol{V} \cdot \hat{\boldsymbol{b}} \\
+q_{\perp} \nabla \cdot \hat{\boldsymbol{b}}=-\frac{1}{3} \nu_{\mathrm{eff}}\left(p_{\perp}-p_{\|}\right), \\
q_{\|}=-\rho \kappa_{\|} \nabla_{\|}\left(\frac{p_{\|}}{\rho}\right) \\
q_{\perp}=-\rho \kappa_{\perp} \nabla_{\|}\left(\frac{p_{\|}}{\rho}\right)+\kappa_{m} \boldsymbol{B} \cdot \nabla \boldsymbol{B},
\end{gathered}
$$

where $\boldsymbol{q}_{\|}=q_{\|} \hat{\boldsymbol{b}}$ and $\boldsymbol{q}_{\perp}=q_{\perp} \hat{\boldsymbol{b}}$ are the heat fluxes parallel to the magnetic field, $\nu_{\text {eff }}$ is the effective pitch-angle scattering rate (including microinstabilities; see $\S 2.3$ and Appendix A3), $\kappa_{\|}$and $\kappa_{\perp}$ are the coefficients of heat conduction, and $\kappa_{m}$ is the coefficient in $q_{\perp}$ due to parallel gradients in the strength of magnetic field (SHD97). The $\kappa_{m}$ component of $q_{\perp}$ that arises because of parallel magnetic field gradients is important for correctly recovering the saturated state for the mirror instability in the fluid limit, where (in steady state) $q_{\|, \perp} \approx 0$ implies that $T_{\|}$is constant along the field line, and $T_{\perp}$ and magnetic pressure are anticorrelated.

Given our closure models, the coefficients for the heat flux are given by

$$
\kappa_{\|}=\frac{8 p_{\|}}{\rho} \frac{1}{\sqrt{8 \pi \frac{p_{\|}}{\rho}} k_{\mathrm{L}}+(3 \pi-8) \nu_{\mathrm{eff}}},
$$

$$
\begin{gathered}
\kappa_{\perp}=\frac{p_{\|}}{\rho} \frac{1}{\sqrt{\frac{\pi}{2} \frac{p_{\|}}{\rho}} k_{\mathrm{L}}+\nu_{\mathrm{eff}}}, \\
\kappa_{m}=\left(1-\frac{p_{\perp}}{p_{\|}}\right) \frac{p_{\perp}}{B^{2}} \kappa_{\perp},
\end{gathered}
$$

where $k_{\mathrm{L}}$ is the parameter that corresponds to a typical wavenumber characterizing Landau damping (see $\S 2$ ). We consider several values of $k_{\mathrm{L}}$ to study the effect of Landau damping on different scales. In particular, we consider $k_{L}=0.5 / \delta z, 0.25 / \delta z$, $0.125 / \delta z$, which correspond to correctly capturing Landau damping on scales of $12 \delta z, 24 \delta z, 48 \delta z$, respectively, where $\delta z=L_{z} / N_{z}$, $L_{z}=1$ for all our runs and $N_{z}$ is the number of grid points used in the $z$-direction (taken be 27 and 54 for low- and high-resolution calculations, respectively). Thus, $k_{\mathrm{L}}=0.25 / \delta z$ corresponds to correctly capturing Landau damping for modes with wavelengths comparable to the size of the box in the low-resolution runs.

The term $\nu_{\text {eff }}$ in equations (42) and (43) is an effective collision frequency thas is equal to the real collision frequency $\nu$ as long as $\mu$ conservation is satisfied. However, when the pressure anisotropy is large enough to drive microinstabilities that break $\mu$ invariance and enhance pitch angle scattering, then there is an increase in the effective collision frequency that decreases the associated conductivities. The expressions for $\nu_{\text {eff }}$ are given in equations (A12), (A15), and (A18) of Appendix A3.

Shearing periodic boundary conditions appropriate to the shearing box are described in HGB95. Excluding $V_{y}$, all variables at the inner $x$ - boundary are mapped to sheared ghost zones at the outer boundary; a similar procedure applies for the inner ghost zones. The variable $V_{y}$ has a jump of $(3 / 2) \Omega L_{x}$ across the box while applying the $x$-shearing boundary conditions, to account for the background shear in $V_{y}$.

\subsection{Numerical Methods}

We have used a version of the ZEUS code modified to include kinetic effects (see Stone \& Norman 1992a, 1992b). ZEUS is a time-explicit, operator-split, finite-difference algorithm on a staggered mesh, i.e., scalars and the diagonal components of second rank tensors are zone centered, while vectors are located at zone faces, and pseudovectors and off-diagonal components of second rank tensors are located at the edges. The location of different variables on the grid is described in more detail in Appendix A1. Appendix A2 describes how we choose the time step $\delta t$ to satisfy the Courant condition (which is modified by pressure anisotropy and heat conduction). We also require that the choice of $\delta t$ maintain positivity of $p_{\|}$and $p_{\perp}$.

Implementation of the shearing box boundary conditions is described in HGB95. One can either apply boundary conditions on the components of $\boldsymbol{B}$ or the EMF. We apply shearing periodic boundary conditions on the EMF to preserve the net vertical flux in the box, although applying boundary conditions directly on $\boldsymbol{B}$ also gives satisfactory results.

Equations (38) and (39) are split into a transport and source step, analogous to the energy equation in the original MHD formalism. The transport step is advanced conservatively, and source step uses central differences in space. It should be noted that in equation (39) the $\nabla \cdot \boldsymbol{q}_{\perp}$ term is not purely diffusive, and it is necessary to carefully treat the magnetic gradient part of $q_{\perp}$ in the transport step for robustness of the code (Appendix A4).

We have carried out a series of tests of our newly added subroutines for evolving anisotropic pressure and parallel heat conduction. We tested the anisotropic conduction routine by initializing a 
"hot" patch in circular magnetic field lines and assessing the extent to which heat remains confined along the field. This is the same test described in detail in I. J. Parrish \& J. M. Stone (2005) and we find good agreement with their results. For the diffusion of a narrow temperature pulse in one dimension, the anisotropic conduction routine also gave results nearly identical to that predicted analytically by the one-dimensional diffusion equation. ${ }^{4}$ Additional tests of the code included linear waves and instabilities in nonrotating anisotropic plasmas, including the Alfvén wave and the firehose and mirror instabilities. For mirror simulations we observe the formation of stationary anticorrelated density and magnetic structures as seen in the hybrid simulations of McKean et al. (1993). For firehose we see the instability with magnetic perturbations developing at small scales but during saturation the perturbations are at larger scales as seen in Quest \& Shapiro (1996).

Finally, the numerical growth rates of the kinetic MRI were compared to the analytic results for different pressure anisotropies, $\left(k_{x}, k_{z}\right)$, collision frequencies, and angles between the magnetic field and $\hat{z}$; we find good agreement with the results of QDH02 and SHQ03. When $k_{\mathrm{L}}=k_{\|}$, the growth rate of the fastest growing mode is within $\sim 3 \%$ of the theoretical prediction. The $B_{\phi}=B_{z}$ MRI simulations show linear growth rates about twice faster than $B_{\phi}=0$, as expected from theory.

\subsection{Shearing Box and Kinetic MHD}

Certain analytic constraints on the properties and energetics of shearing box simulations have been described in HGB95. These constraints serve as a useful check on the numerical simulations. Here we mention the modifications to these constraints in KMHD. Conservation of total energy in the shearing box gives

$$
\frac{\partial}{\partial t} \Gamma=\frac{3}{2} \Omega L_{x} \int_{x} d A\left\{\rho V_{x} \delta V_{y}-\left[1-\frac{4 \pi\left(p_{\|}-p_{\perp}\right)}{B^{2}}\right] \frac{B_{x} B_{y}}{4 \pi}\right\}
$$

where $\delta V_{y}=V_{y}+(3 / 2) \Omega x$, and $\Gamma$ is the total energy given by

$$
\Gamma=\int d^{3} x\left[\rho\left(\frac{V^{2}}{2}+\phi\right)+\frac{p_{\|}}{2}+p_{\perp}+\frac{B^{2}}{8 \pi}\right]
$$

where $\phi=-3 / 2 \Omega^{2} x^{2}$ is the tidal effective potential about $R_{0}$. Equation (45) states that the change in the total energy of the shearing box is due to work done on the box by the boundaries. Notice that there is an anisotropic pressure contribution to the work done on the box. Equation (29) in Balbus \& Hawley (1998) for conservation of angular momentum in cylindrical geometry is also modified because of the anisotropic pressure and is given by

$$
\begin{aligned}
\frac{\partial}{\partial t}\left(\rho R V_{\phi}\right)+\nabla & \cdot\left\{\rho V_{\phi} \boldsymbol{V} R-\frac{B_{\phi}}{4 \pi}\left[1-\frac{4 \pi\left(p_{\|}-p_{\perp}\right)}{B^{2}}\right] \boldsymbol{B}_{p} R\right. \\
+ & \left.\left(p_{\perp}+\frac{B_{p}^{2}}{8 \pi}\right) \hat{\phi} R\right\}=0,
\end{aligned}
$$

\footnotetext{
${ }^{4}$ For details of the test and error analysis, see http://w3.pppl.gov/ psharma/ cartesian/1Dheatdiffusion.
}

where $\boldsymbol{B}_{\boldsymbol{p}}=B_{R} \hat{\boldsymbol{R}}+B_{z} \hat{\mathbf{z}}$ is the poloidal field. We can calculate the level of angular momentum transport in our simulations by measuring the stress tensor given by

$$
W_{x y}=\rho V_{x} \delta V_{y}-\frac{B_{x} B_{y}}{4 \pi}+\frac{\left(p_{\|}-p_{\perp}\right)}{B^{2}} B_{x} B_{y}
$$

Note that the stress tensor has an additional contribution due to pressure anisotropy. One can define a dimensionless stress via Shakura and Sunyaev's $\alpha$ parameter by

$$
\alpha \equiv \frac{W_{x y}}{P_{0}}=\alpha_{R}+\alpha_{M}+\alpha_{A},
$$

where $\alpha_{\mathrm{R}}, \alpha_{\mathrm{M}}, \alpha_{A}$ are the Reynolds, Maxwell and anisotropic stress parameters, respectively. As in HGB95 we normalize the stress using the initial pressure to define an $\alpha$ parameter.

\subsection{Shearing Box Parameters and Initial Conditions}

The parameters for our baseline case have been chosen to match the fiducial run Z4 of HGB95. The simulation box has a radial size $L_{x}=1$, azimuthal size $L_{y}=2 \pi$, and vertical size $L_{z}=1$. The sound speed $V_{s}=(p / \rho)^{1 / 2}=L_{z} \Omega$, so that the vertical size is about a disk scale height (although it is an unstratified box). The pressure is assumed to be isotropic initially, with $p_{0}=\rho_{0} V_{s}^{2}=$ $10^{-6}$ and $\rho_{0}=1$. All of our simulations start with a vertical field with $\beta=8 \pi p_{0} / B_{0}^{2}=400$. The fastest growing MRI mode for this choice of parameters is reasonably well resolved. We consider two different numerical resolutions: $27 \times 59 \times 27$, and $54 \times 118 \times 54$. Perturbations are introduced as initially uncorrelated velocity fluctuations. These fluctuations are randomly and uniformly distributed throughout the box. They have a mean amplitude of $|\delta V|=10^{-3} V_{s}$.

\section{RESULTS}

The important parameters for our simulations are listed in Table 1. Each simulation is labeled by $Z$ (for the initial $B_{z}$ field), and $l$ and $h$ represent low $(27 \times 59 \times 27)$ and high $(54 \times 118 \times 54)$ resolution runs, respectively. We also include low- and highresolution MHD runs for comparison with the kinetic calculations (labeled by $Z M$ ). Our models for heat conduction and pressure isotropization have several parameters: $k_{\mathrm{L}}$, the typical wavenumber for Landau damping used in the heat flux (eqs. [13] and [14]), and $\xi$, the parameter that forces the pressure anisotropy to be limited by $p_{\perp} / p_{\|}-1<2 \xi / \beta_{\perp}$ (representing pitch angle scattering due to small-scale mirror modes; eq. [33]). All of our calculations except $Z l 8, Z l 1$, and $Z h 1$ also use the ion cyclotron scattering "hard wall" from equation (34). In addition to these model parameters, Table 1 also lists the results of the simulations, including the volume- and time-averaged magnetic and kinetic energies, and Maxwell, Reynolds, and anisotropic stresses. As Table 1 indicates, the results of our simulations depend quantitatively — although generally not qualitatively — on the microphysics associated with heat conduction and pressure isotropization. Throughout this section we use single brackets $\langle f\rangle$ to denote a volume average of quantity $f$; we use double brackets $\langle\langle f\rangle\rangle$ to denote a volume and time average in the saturated turbulent state, from orbit 5 onward.

\subsection{Fiducial Run}

We have selected run $Z l 4$ as our fiducial model to describe in detail. This model includes isotropization by ion cyclotron instabilities and mirror modes, with the former dominating (for $\xi=3.5$; see $\S 2.2 .2$ ) except at early times. The conductivity is determined 
TABLE 1

Simulation Parameters

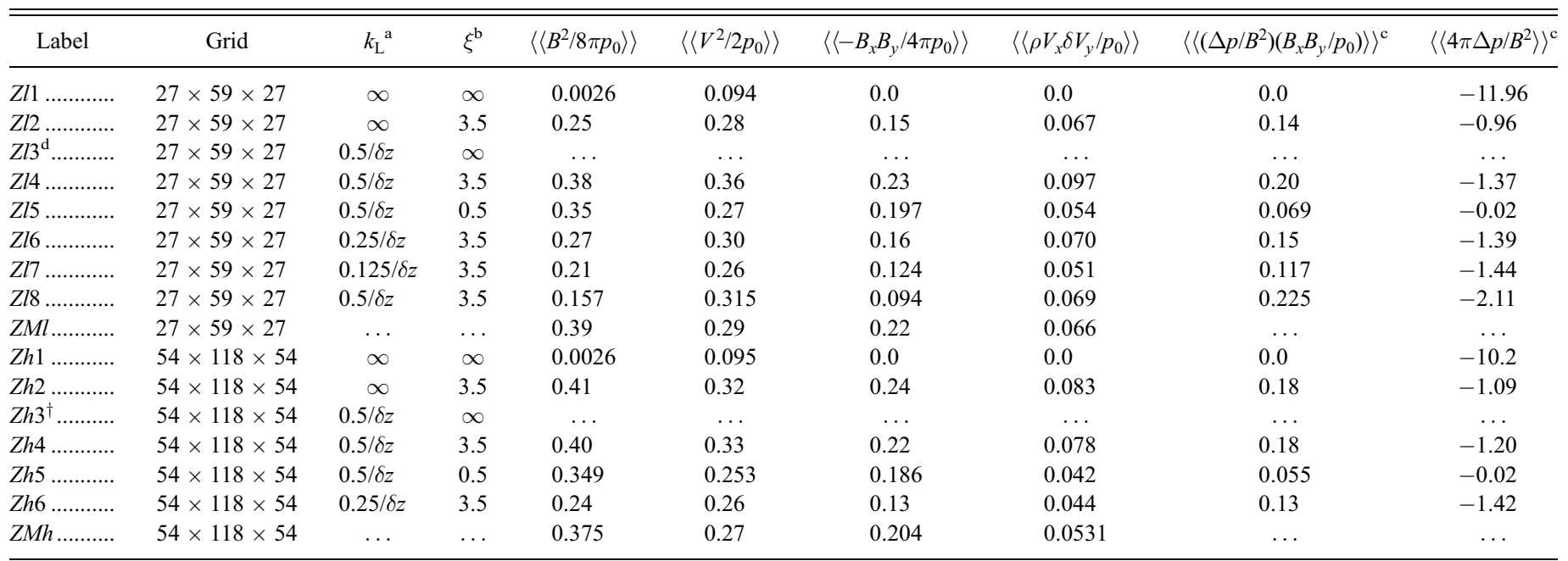

Notes.-Vertical field simulation with initial $\beta=400$. $Z$ indicates that all simulations start with a vertical field, the letters $l$ and $h$ indicate low- and high-resolution runs respectively. $Z l 4$ is the fiducial run. $Z l 1$ and $Z h 1$ are the runs in CGL limit. $Z M l$ and $Z M h$ are the MHD runs. Double angle brackets denote a time and space average taken from 5-20 orbits.

${ }^{a}$ Wavenumber parameter used in Landau closure for parallel heat conduction (eqs. [13] and [14]).

${ }^{\mathrm{b}}$ Imposed limit on pressure anisotropy for pitch angle scattering due to mirror instability (eq. [33]). Excluding $Z l 1, Z h 1$, and $Z l 8$ all of these calculations also use a pressure anisotropy limit due to the ion cyclotron instability (eq. [34]).

c $\Delta p=\left(p_{\|}-p_{\perp}\right)$.

${ }^{\mathrm{d}}$ Run for only $\approx$ four orbits, at which point the time step becomes very small because regions of large pressure anisotropy are created (see $\S 4.4$ ).

by $k_{\mathrm{L}}=0.5 / \delta z$ which implies that modes with wavelengths $\sim 12 \delta z \sim L_{z} / 2$ are damped at a rate consistent with linear theory.

Figures $2-4$ show the time evolution of various physical quantities for run $Z l 4$. The early linear development of the instability is similar to that in MHD, with the field growing exponentially in time. The key new feature is the simultaneous exponential growth of pressure anisotropy $\left(p_{\perp}>p_{\|}\right)$as a result of $\mu$ conservation (up to two orbits in Fig. 4). As described in $\S 2.1$, this pressure anisotropy tends to stabilize the MRI modes and shut off the

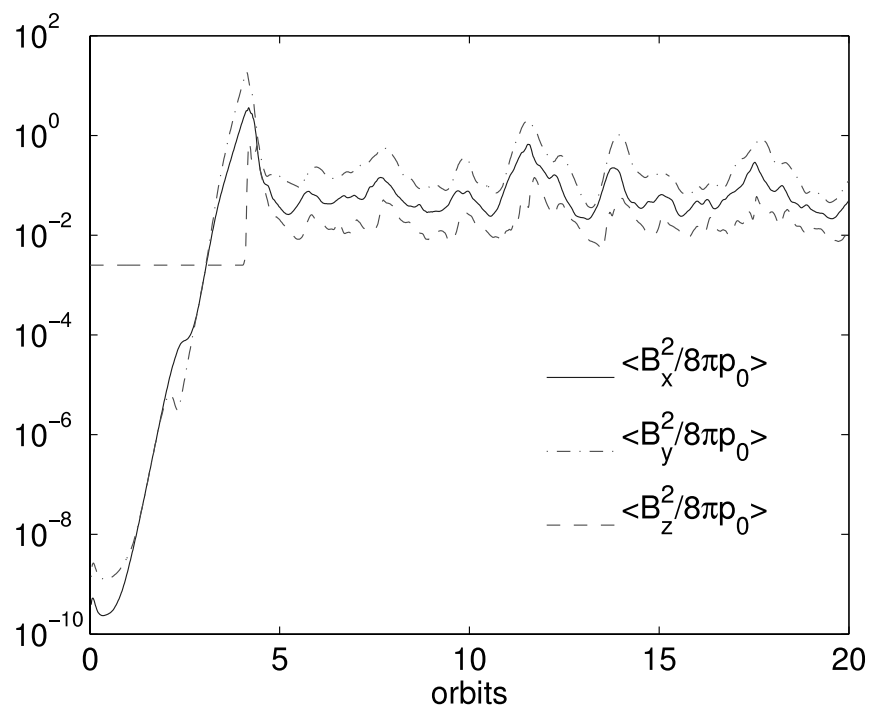

FIG. 2.-Time evolution of volume-averaged magnetic energy for the fiducial run Zl4. Time is given in number of orbits. There is a small decrease in the magnetic energy at $\approx$ to orbits when the pressure anisotropy is sufficient to stabilize the fastest growing mode. However, small-scale kinetic instabilities limit the magnitude of the pressure anisotropy, allowing the magnetic field to continue to amplify. As in MHD, there is a channel phase that breaks down into turbulence at $\approx$ four orbits. growth of the magnetic field. Indeed, in simulations that do not include any isotropization of the pressure tensor, we find that all MRI modes in the box are stabilized by the pressure anisotropy and the simulation saturates with the box filled with small amplitude anisotropic Alfvén waves (see Fig. 6). This highlights the fact that, unlike in MHD, the MRI is not an exact nonlinear solution in kinetic theory. However, the pressure anisotropy required to stabilize all MRI modes exceeds the pressure anisotropy at which pitch angle scattering due to mirror and ion cyclotron instabilities become important. This takes place at about orbit 2 in run $Z l 4$ (see the small "dip" in the growth of B in Fig. 2), at which point the pressure anisotropy is significantly reduced and the magnetic field is able to grow to nonlinear amplitudes.

The nonlinear saturation at orbit $\sim 5$ appears qualitatively similar to that in MHD, and may occur via analogues of the parasitic instabilities described by Goodman \& Xu (1994). The channel solution is, however, much more extreme in KMHD than MHD (the maximum $B^{2}$ in Fig. 2 is approximately an order of magnitude larger than in analogous MHD runs). After saturation, the magnetic and kinetic energies in the saturated state are comparable in KMHD and MHD (see Table 1). This is essentially because the pitch angle scattering induced by the kinetic microinstabilities acts to isotropize the pressure, enforcing a degree of MHD-like dynamics on the collisionless plasma.

Figure 3 and Table 1 show the various contributions to the total stress. As in MHD, the Reynolds stress is significantly smaller than the Maxwell stress. In kinetic theory, however, there is an additional component to the stress due to the anisotropic pressure (eq. [47]). In the saturated state, we find that the Maxwell stress is similar in KMHD and MHD, but that the anisotropic stress itself is comparable to the Maxwell stress. Expressed in terms of an $\alpha$ normalized to the initial pressure, our fiducial run $Z l 4$ has $\alpha_{M}=$ $0.23, \alpha_{\mathrm{R}}=0.097$, and $\alpha_{A}=0.2$, indicating that stress due to pressure anisotropy is dynamically important.

Nearly all physical quantities in Figures $2-4$ reach an approximate statistical steady state. The exceptions are that $p_{\|}$and $p_{\perp}$ 

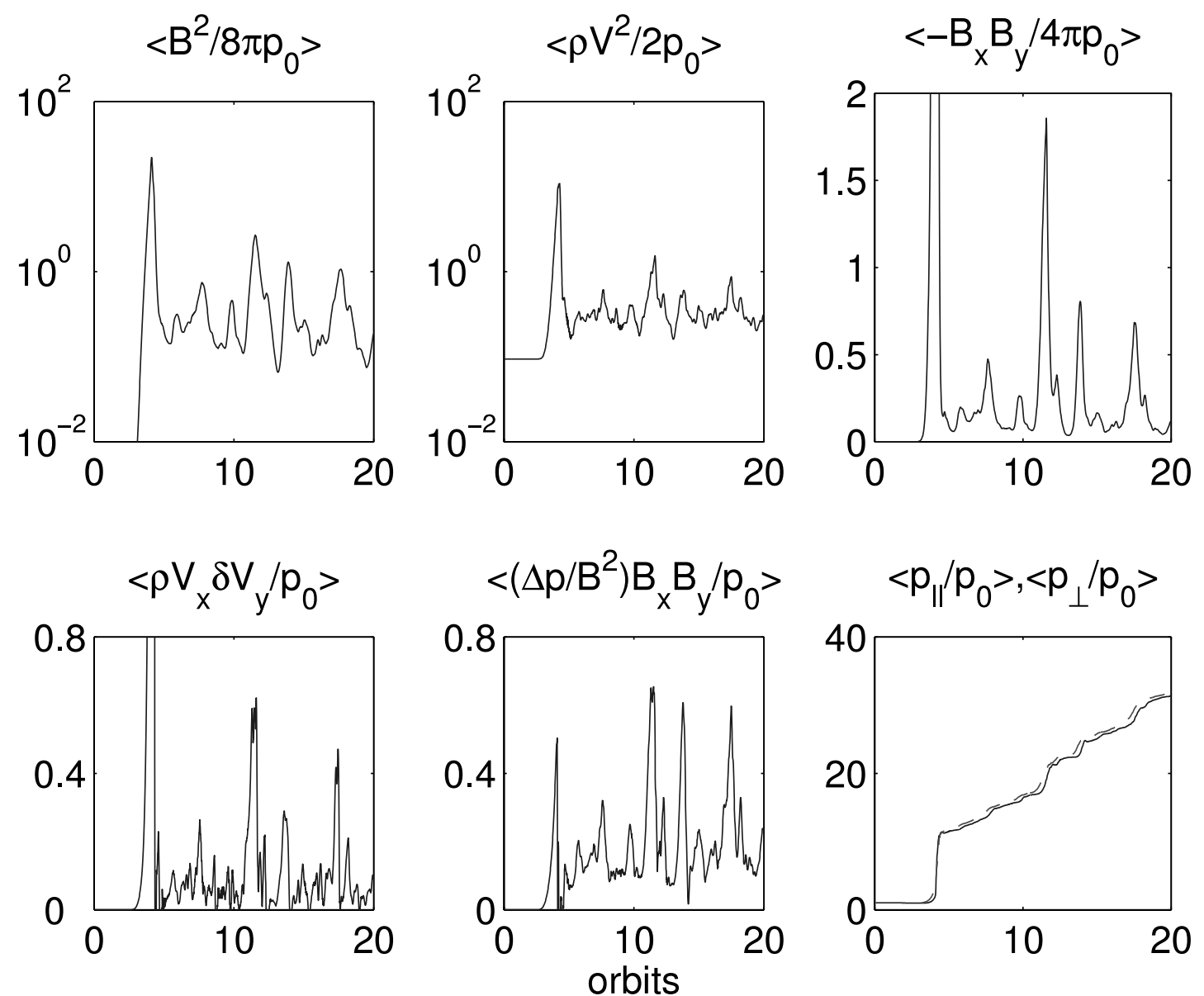

Fig. 3. - Time evolution of volume-averaged magnetic and kinetic energies; Maxwell, Reynolds, and anisotropic stress; and pressure (solid line, $p_{\|}$; dashed line, $\left.p_{\perp}\right)$ for the fiducial model $Z l 4$. Time is given in orbits and all quantities are normalized to the initial pressure $p_{0} ; \delta V_{y}=V_{y}+(3 / 2) \Omega x$ and $\Delta p=\left(p_{\|}-p_{\perp}\right)$.

$<4 \pi\left(p_{\|}-p_{\perp}\right) / B^{2}>$

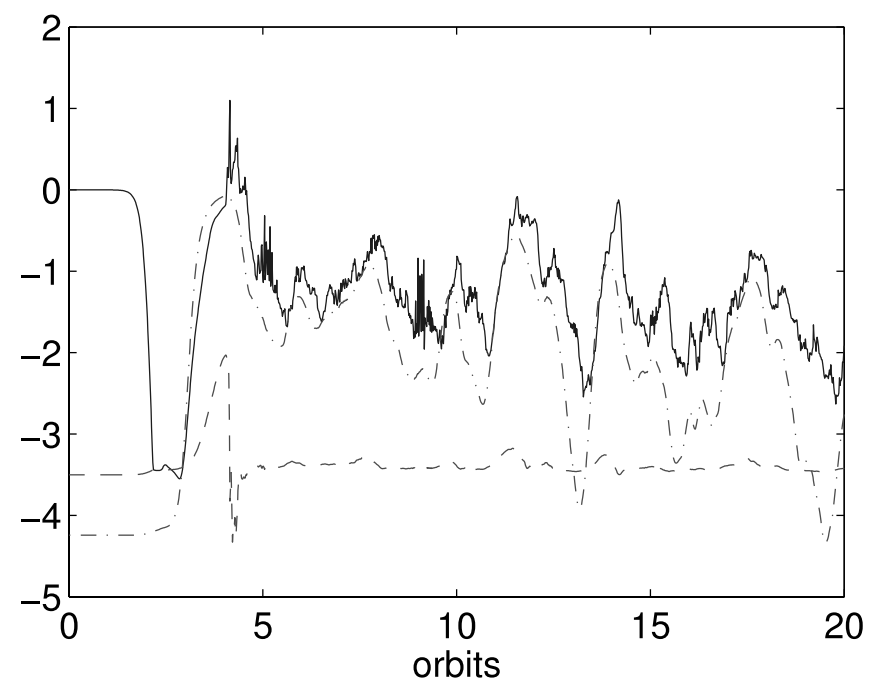

FIG. 4.-Time evolution of volume-averaged pressure anisotropy [solid line, $\left.4 \pi\left(p_{\|}-p_{\perp}\right) / B^{2}\right]$ for model Zl4. Also plotted are the "hard wall" limits on the pressure anisotropy due to the ion cyclotron (dot-dashed line) and mirror instabilities (dashed line). Ion cyclotron scattering is generally more efficient in the steady state. The limits on pressure anisotropy are applied at each grid point, while this figure is based on volume-averaged quantities. increase steadily in time because the momentum flux on the boundaries does work on the system (eq. [45]), which is eventually converted to heat in the plasma by artificial viscosity and there is no cooling (the same is true in HGB95's MHD simulations). Because of the steadily increasing internal energy and approximately fixed $B^{2}$ (although with large fluctuations), the plasma $\beta$ shows a small secular increase from orbits $5-20$ (a factor of $\approx 3$ increase, although with very large fluctuations due to the large fluctuations in magnetic energy). Figure 4 shows the pressure anisotropy thresholds due to the ion cyclotron and mirror instabilities, in addition to the volume-averaged pressure anisotropy in run Zl4. From equation (34), the ion cyclotron threshold is expected to scale as $\beta_{\|}^{1 / 2}$, which is reasonably consistent with the trend in Figure 4. The actual pressure anisotropy in the simulation shows a small increase in time as well, although less than that of the ion cyclotron threshold. These secular changes in $\beta$ and $\Delta p$ are a consequence of the increasing internal energy in the shearing box, and are probably not realistic. In a global disk, we expect that - except perhaps near the inner and outer boundaries$\beta$ will not undergo significant secular changes in time. In a small region of a real disk in statistical equilibrium, the heating would be balanced by radiation or by cooler plasma entering at large $R$ and hotter plasma leaving at small $R$.

It is interesting to note that in Figure 4, the pressure anisotropy $\left(4 \pi \Delta p / B^{2}\right)$ is closely tied to the ion cyclotron threshold at times when $B^{2}$ is rising (which corresponds to the channel solution reemerging). Increasing $B$ leads to a pressure anisotropy with $p_{\perp}>p_{\|}$by $\mu$ conservation. At the same time, the ion cyclotron 


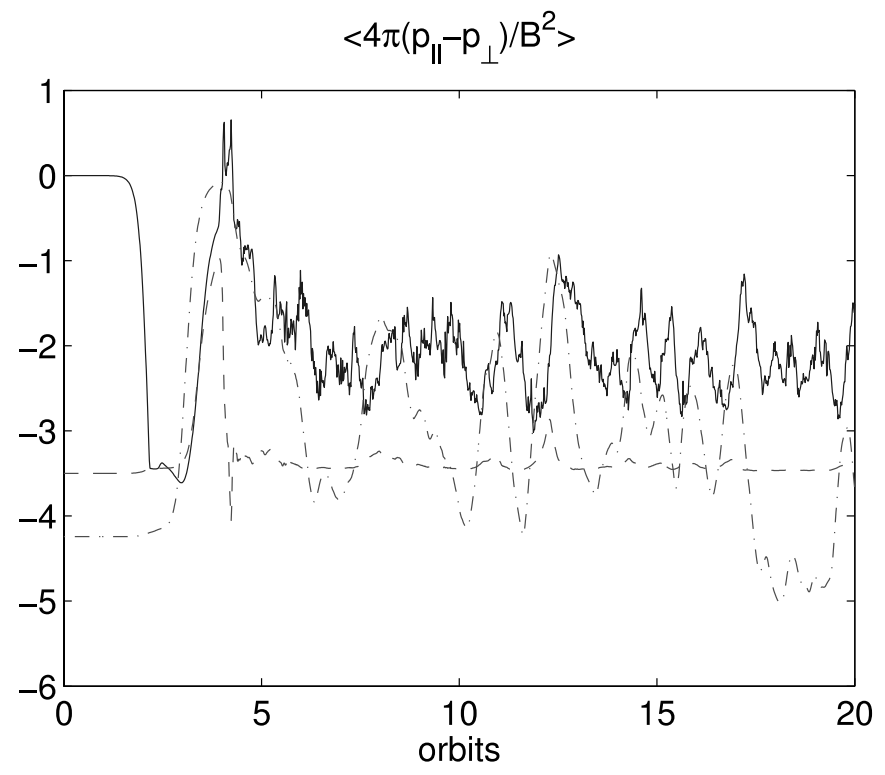

FIG. 5.-Time evolution of volume-averaged pressure anisotropy [solid line, $\left.4 \pi\left(p_{\|}-p_{\perp}\right) / B^{2}\right]$ for model Zl8. Also plotted are the "hard wall" limits on the pressure anisotropy due to the ion cyclotron (dot-dashed line) and mirror instabilities (dashed line), although the ion cyclotron scattering limit is not applied in this simulation. The volume-averaged pressure anisotropy saturates at smaller anisotropy than the mirror threshold at $\xi=3.5$, which is the only limit on pressure anisotropy used.

threshold $(\sim \sqrt{\beta})$ decreases and thus the threshold is encountered, which limits the anisotropy. When $B$ is decreasing, however, we do not find the same tight relationship between the pressure anisotropy and the imposed threshold. Figure 4 clearly indicates that in our fiducial simulation pitch angle scattering is dominated by the ion cyclotron threshold. For comparison, Figure 5 shows the pressure anisotropy and thresholds for run $Z l 8$, which is iden- tical to the fiducial run except that the ion cyclotron threshold is not used and the only scattering is due to the mirror threshold. In this case, the saturated pressure anisotropy is somewhat larger than in the fiducial run, but the pressure anisotropy is not tied to the mirror threshold.

Table 2 gives the mean, standard deviation, and standard error in the mean, for various quantities in the saturated portion of the fiducial simulation. The standard errors are estimated by taking into account the finite correlation time for the physical quantities in the simulation, as described in Appendix A5. In many cases, the deviations are significantly larger than the mean. As in MHD, we find that the magnetic energy is dominated by the $y$-component, which is about a factor of 3 larger than the $x$-component; the vertical component is smaller yet. The radial and azimuthal kinetic energy fluctuations are comparable, while the vertical component is smaller. We also find that, as in MHD, the perturbed kinetic and magnetic energies are not in exact equipartition: the magnetic energy is consistently larger. Table 2 also shows the mean and deviations for $\left\langle p_{\perp} / B\right\rangle$ and $\left\langle p_{\|} B^{2} / \rho^{2}\right\rangle$. Because of pitch angle scattering $\mu=\left\langle p_{\perp} / B\right\rangle$ is no longer conserved. Here $\left\langle p_{\|} B^{2} / \rho^{2}\right\rangle$ varies both because of heat conduction and pitch angle scattering.

The pressure anisotropy in our fiducial run saturates at $4 \pi\left(p_{\perp}-p_{\|}\right) / B^{2} \approx 1.5$. By contrast, the threshold for the mirror instability is $4 \pi\left(p_{\perp}-p_{\|}\right) / B^{2}=0.5$. This implies that the model is unstable to generating mirror modes. However, the mirror modes that can be excited at this level of anisotropy do not violate $\mu$ conservation and thus do not contribute to pitch angle scattering $(\S 2.2 .2)$. They can in principle isotropize the plasma in a volume-averaged sense by spatially redistributing plasma into magnetic wells (e.g., Kivelson \& Southwood 1996). This saturation mechanism can be calculated using our kinetic-MHD code and was in fact one of our test problems (for a uniform plasma). It does not appear to be fully efficient in the saturated state of our turbulent disk simulations, even at the highest resolutions we have run.

TABLE 2

Statistics for Model $Z l 4$

\begin{tabular}{|c|c|c|c|c|c|}
\hline Quantity $f$ & $\langle\langle f\rangle\rangle$ & $\left\langle\left\langle\delta f^{2}\right\rangle\right\rangle^{1 / 2}$ & $\left(\tau_{\text {int }} / T\right)^{1 / 2}\left\langle\left\langle\delta f^{2}\right\rangle\right\rangle^{1 / 2}$ & $\min (f)$ & $\max (f)$ \\
\hline$B_{x}^{2} / 8 \pi p_{0}$ & 0.083 & 0.092 & 0.016 & 0.021 & 0.662 \\
\hline 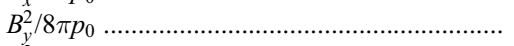 & 0.276 & 0.318 & 0.048 & 0.036 & 1.987 \\
\hline 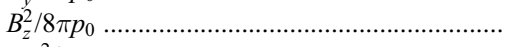 & 0.021 & 0.017 & 0.0025 & 0.0032 & 0.144 \\
\hline 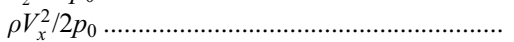 & 0.102 & 0.094 & 0.014 & 0.0184 & 0.63 \\
\hline 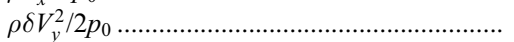 & 0.125 & 0.079 & 0.0127 & 0.715 & 0.0264 \\
\hline 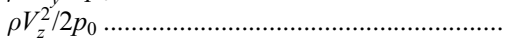 & 0.037 & 0.034 & 0.0032 & 0.008 & 0.348 \\
\hline 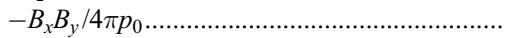 & 0.229 & 0.277 & 0.0434 & 0.037 & 1.856 \\
\hline 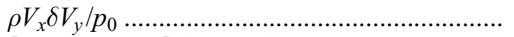 & 0.097 & 0.113 & 0.0147 & -0.072 & 0.6211 \\
\hline 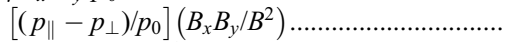 & 0.198 & 0.129 & 0.0178 & 0.017 & 0.654 \\
\hline 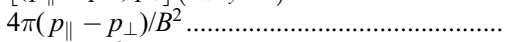 & -1.366 & 0.51 & 0.098 & -2.632 & -0.083 \\
\hline 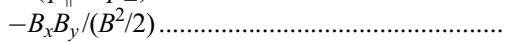 & 0.5895 & 0.1043 & 0.0067 & 0.3744 & 0.8611 \\
\hline 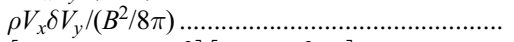 & 0.3323 & 0.2725 & 0.017 & -0.5307 & 1.2704 \\
\hline$\left[4 \pi\left(p_{\|}-p_{\perp}\right) / B^{2}\right]\left[B_{x} B_{y} /\left(B^{2} / 2\right)\right] \ldots \ldots \ldots \ldots \ldots \ldots \ldots \ldots \ldots$ & 0.7356 & 0.3718 & 0.0714 & 0.032 & 1.807 \\
\hline 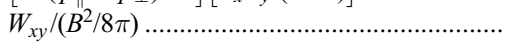 & 1.6574 & 0.6598 & 0.084 & 0.4364 & 3.7159 \\
\hline 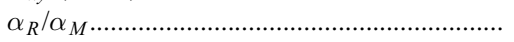 & 0.5357 & 0.3975 & 0.024 & -0.9105 & 2.084 \\
\hline 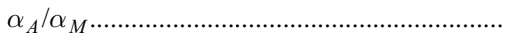 & 1.2287 & 0.5504 & 0.119 & 0.0854 & 2.7243 \\
\hline 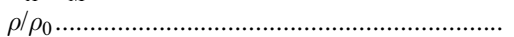 & 0.99935 & $2.3 \times 10^{-5}$ & $1.1 \times 10^{-5}$ & 0.9993 & 0.9994 \\
\hline 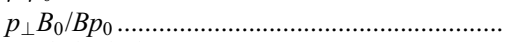 & 3.557 & 1.665 & $\ldots{ }^{\mathrm{a}}$ & 1.1178 & 7.929 \\
\hline$p_{\|} B^{2} \rho_{0}^{2} / \rho^{2} B_{0}^{2} p_{0}$ & $3.144 \times 10^{3}$ & $3.49 \times 10^{3}$ & $\cdots$ & 585.4 & $1.993 \times 10^{4}$ \\
\hline
\end{tabular}

NotE.-Double angle brackets denote a time and space average taken from 5-20 orbits.

${ }^{a}$ We calculate the error using the autocorrelation time only for quantities that saturate to a steady state after five orbits. Estimate for correlation time $\tau_{\text {int }}$ is based on the discussion in W. Nevins et al. (2006, in preparation). $p_{\perp}$ and $p_{\|}$show a secular growth with time, so this way of expressing them as an average and an error is not applicable. 

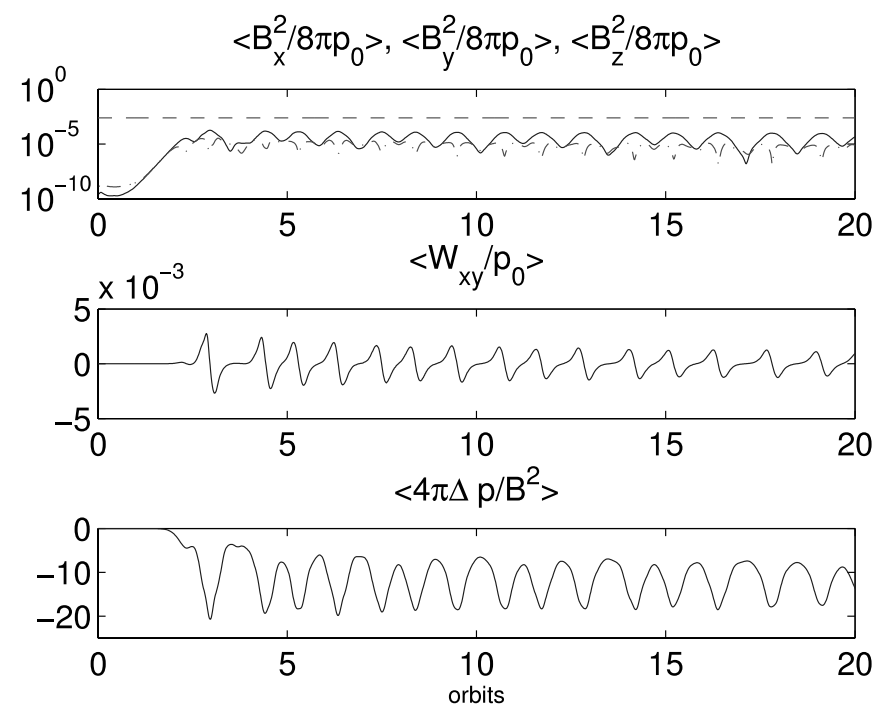

Fig. 6.-Time evolution of volume-averaged magnetic energy (dashed line, $B_{z}^{2} / 8 \pi p_{0} ;$ solid line, $B_{x}^{2} / 8 \pi p_{0} ;$ dot-dashed line, $\left.B_{y}^{2} / 8 \pi p_{0}\right)$, total stress $\left(W_{x v} / p_{0}\right)$ in units of $10^{-3}$, and pressure anisotropy for model $Z l 1$. Time is given in orbits and all quantities are normalized to the initial pressure $p_{0} ; \delta V_{y}=V_{y}+(3 / 2) \Omega x$ and $\Delta p=\left(p_{\|}-p_{\perp}\right)$. In this calculation there is no heat conduction and no isotropization of the pressure tensor. All resolved MRI modes are thus stabilized by pressure anisotropy and the "saturated" state is linear anisotropic Alfvén waves with no net angular momentum transport.

In the next few sections we compare the fiducial simulation described above with variations in the pitch angle scattering model and the parallel conductivity. A comparison of the total stress in all of our simulations is shown in Figure 7.

\subsection{The Double Adiabatic Limit}

Simulations $Z l 1$ and $Z h 1$ are simulations in the double adiabatic limit (no heat conduction), with no limit on the pressure anisotropy imposed. In this limit both $\mu=\left\langle p_{\perp} / B\right\rangle$ and $\left\langle p_{\|} B^{2} / \rho^{2}\right\rangle$ are conserved. Figure 6 shows volume averages of various quantities as a function of time for the run $Z l 1$. These calculations are very different from the rest of our results and show saturation at very low amplitudes $\left(\delta B^{2} / B^{2} \approx 0.04\right)$. In the saturated state, the box is filled with shear modified anisotropic Alfvén waves and all physical quantities are oscillating in time. The total stress is also oscillatory with a vanishing mean, resulting in negligible transport. In these calculations, the pressure anisotropy grows to such a large value that it shuts off the growth of all of the resolved MRI modes in the box. Table 1 shows that $\left\langle\left\langle 4 \pi\left(p_{\|}-\right.\right.\right.$ $\left.\left.\left.p_{\perp}\right) / B^{2}\right\rangle\right\rangle$ saturates at -11.96 and -10.2 for the low- and highresolution runs, respectively (although the normalized pressure anisotropy $\left\langle\left\langle\left(p_{\|}-p_{\perp}\right) / p_{\|}\right\rangle\right\rangle \approx-0.07$ is quite small). This is much larger than the anisotropy thresholds for pitch angle scattering described in $\S 2$. As a result, we do not expect these cases to be representative of the actual physics of collisionless disks. These cases are of interest, however, in supporting the predictions of the linear theory with anisotropic initial conditions considered in $\S 2.1$, and in providing a simple test for the simulations. They also highlight the central role of pressure isotropization in collisionless dynamos.

\subsection{Varying Conductivity}

We have carried out a series of simulations with different conductivities defined by the parameter $k_{\mathrm{L}}$. Simulations $Z l 2$ and $Z h 2$

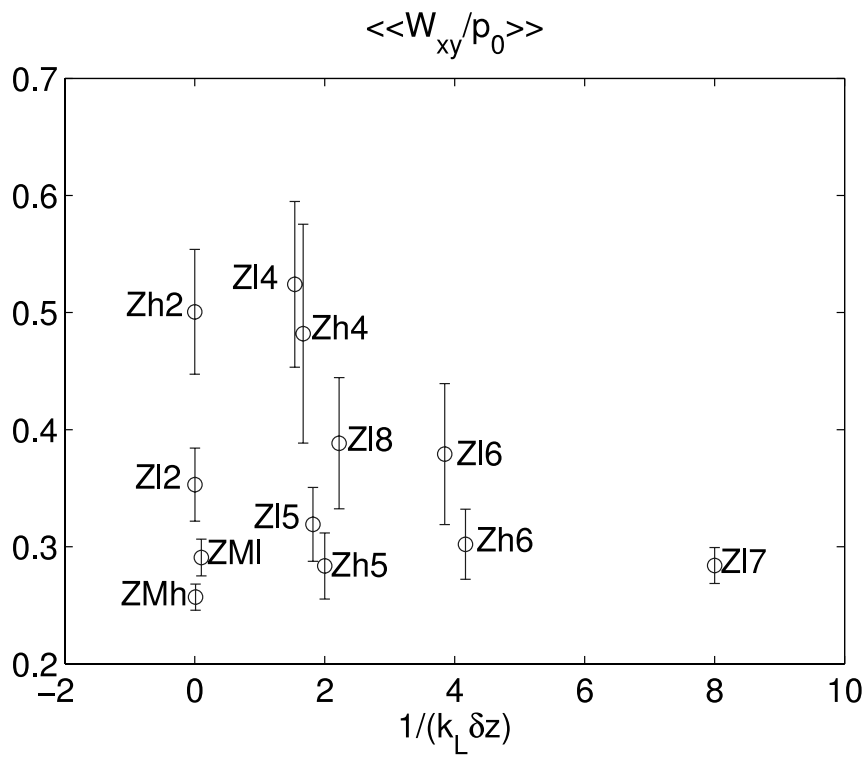

FIG. 7.- Space and time average of the total stress $\left\langle\left\langle W_{x y} / p_{0}\right\rangle\right\rangle$ vs. $1 /\left(k_{\mathrm{L}} \delta z\right)$ for different runs. Error bars shown are based on estimates of the correlation time of the fluctuations described in W. Nevins (2006, in preparation).

are in the CGL limit with vanishing parallel heat conduction, but with the same limits on pressure anisotropy as the fiducial model. Simulations $Z 6$ use $k_{\mathrm{L}} \delta z=0.25$ while run $Z l 7$ uses $k_{\mathrm{L}}=0.125 / \delta z$. Both of these are smaller than the value of $k_{\mathrm{L}} \delta z=0.5$ in the fiducial run, which implies a larger conductivity. Figure 7 shows that the total stress varies by about a factor of 2 depending on the conductivity and resolution. Simulations with larger conductivity tend to have smaller saturation amplitudes and stresses. This could be because larger conductivity implies more rapid Landau damping of slow and fast magnetosonic waves. In all cases, however, the anisotropic stress is comparable to the Maxwell stress as in the fiducial run. Until a more accurate evaluation is available of the heat fluxes for modes of all wavelengths in the simulation simultaneously (either by a more complete evaluation of the nonlocal heat fluxes, eqs. [11] and [12], or even by a fully kinetic MHD code that directly solves eq. [5]), it is difficult to ascertain which value of the conductivity best reflects the true physics of collisionless disks.

\subsection{Different Pitch Angle Scattering Models}

In this section we consider variations in our model for pitch angle scattering by high-frequency waves. All of these calculations utilize $k_{\mathrm{L}}=0.5 / \delta z$. We note again that the appropriate pitch angle scattering model remains somewhat uncertain, primarily because of uncertainties in the nonlinear saturation of longwavelength $\mu$-conserving mirror modes. The calculations reported here cover what we believe is a plausible range of models.

Models $Z l 5$ and $Z h 5$ place a more stringent limit on the allowed pressure anisotropy, taking $\xi=0.5$ in equation (33). This corresponds to the threshold of the mirror instability. Not surprisingly, this simulation is the most "MHD-like" of our calculations, with magnetic and kinetic energies and Maxwell stresses that are quite similar to those in MHD. Even with this stringent limit, however, the anisotropic stress is $\approx \frac{1}{3}$ of the Maxwell stress. It is also interesting to note that although the dimensionless pressure anisotropy is quite small $\left\langle\left\langle 4 \pi\left(p_{\|}-p_{\perp}\right) / B^{2}\right\rangle\right\rangle \approx-0.02$, the dimensionless anisotropic stress $\left\langle\left\langle 4 \pi\left(p_{\|}-p_{\perp}\right) / B^{2} \times B_{x} B_{y} / p_{0}\right\rangle\right\rangle \approx$ 0.07 is significantly larger (and larger than Reynolds stress) 
TABLE 3

Simulations with an Explicit Collision Term

\begin{tabular}{|c|c|c|c|c|c|c|}
\hline$\nu / \Omega$ & $\left\langle\left\langle 4 \pi \Delta p / B^{2}\right\rangle\right\rangle$ & $\left\langle\left\langle-B_{x} B_{y} / 4 \pi p_{0}\right\rangle\right\rangle$ & $\left(\left\langle\left\langle\rho V_{x} \delta V_{y} / p_{0}\right\rangle\right\rangle\right.$ & $\left\langle\left\langle\left(\Delta p / B^{2}\right)\left(B_{x} B_{y} / p_{0}\right)\right\rangle\right\rangle^{\mathrm{a}}$ & $\alpha_{A} / \alpha_{M}$ & $\alpha_{A} / \alpha_{A}(\nu=0)$ \\
\hline 0 & -1.41 & 0.18 & 0.082 & 0.196 & 1.09 & 1 \\
\hline 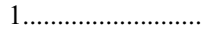 & -1.47 & 0.152 & 0.072 & 0.173 & 1.14 & 0.88 \\
\hline 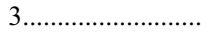 & -1.43 & 0.178 & 0.08 & 0.181 & 1.02 & 0.92 \\
\hline $10 \ldots \ldots \ldots \ldots \ldots . .$. & -1.35 & 0.165 & 0.071 & 0.159 & 0.96 & 0.81 \\
\hline $20 \ldots \ldots \ldots \ldots \ldots \ldots$ & -1.24 & 0.174 & 0.070 & 0.136 & 0.78 & 0.69 \\
\hline $30 \ldots \ldots \ldots \ldots \ldots$ & -1.01 & 0.213 & 0.070 & 0.113 & 0.53 & 0.58 \\
\hline 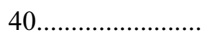 & -0.87 & 0.239 & 0.070 & 0.095 & 0.4 & 0.48 \\
\hline $100 \ldots \ldots \ldots \ldots \ldots \ldots$ & -0.43 & 0.223 & 0.06 & 0.032 & 0.14 & 0.16 \\
\hline
\end{tabular}

Noте.-Double angle brackets denote a time and space average taken from 5-20 orbits.

${ }^{\mathrm{a}} \Delta p=\left(p_{\|}-p_{\perp}\right)$.

because of correlations between the pressure anisotropy and field strength.

As a test of how large a collisionality is needed for the results of our kinetic simulations to rigorously approach the MHD limit, we have carried out a series of simulations including an explicit collisionality $\nu$ and varying its magnitude relative to the disk frequency $\Omega$. Our results are summarized in Table 3 and Figure 8. In these simulations we start with initial conditions determined by the saturated turbulent state of our fiducial run $Z l 4$, but with an explicit collision frequency (in addition to the scattering models described in $\S 2.3$ ). Figure 8 shows that for $\nu / \Omega \lesssim 20$, the results are very similar to the collisionless limit. For larger collision frequencies the anisotropic stress is reduced and the simulations quantitatively approach the MHD limit. These results are similar to those obtained by SHQ03, who found that in linear calculations the MHD limit for modes with $k \sim \Omega / v_{\mathrm{A}}$ is approached when $\nu \gtrsim \sqrt{\beta} \Omega$.

To consider the opposite limit of lower collisionality, run Zl8 places a less stringent limit on the allowed pressure anisotropy, taking $\xi=3.5$ in equation (33) and ignoring the limit set by the ion cyclotron instability in equation (34). The results of this calculation are not physical but are useful for further clarifying the relative importance of the Maxwell and anisotropic stresses as a function of the pitch angle scattering rate. In $Z l 8$, the saturated

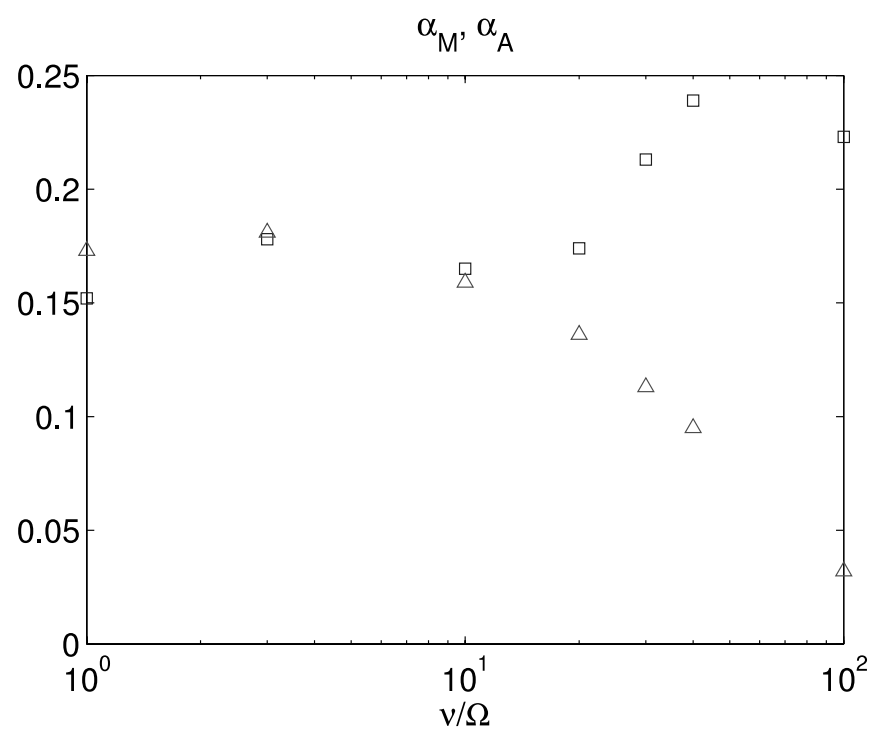

FIG. 8.-Maxwell ( $\alpha_{\mathrm{M}}$, squares $)$ and anisotropic $\left(\alpha_{A}\right.$, triangles $)$ stress plotted against the collision frequency normalized to rotation frequency $(\nu / \Omega)$. Transition to MHD occurs for $\nu / \Omega \gtrsim 30$ (see Table 3). magnetic energy and Maxwell stress are lower than in all of our other calculations (excluding the double adiabatic models described in $\S 4.2$ ). Interestingly, however, the total stress is comparable to that in the other calculations (Fig. 7) because the anisotropic stress is $\approx 2.4$ times larger than the Maxwell stress (Table 1). As discussed briefly in $\S 4.1$, the pressure anisotropy in this simulation is not simply set by the applied mirror pitch angle scattering threshold (see Fig. 5). It is possible that resolved mirror modes contribute to decreasing the volume-averaged pressure anisotropy (but see below).

Finally, in models $Z 3$ we include parallel heat conduction but do not limit the pressure anisotropy. In these calculations, we expect to be able to resolve the long-wavelength $\mu$-conserving mirror modes that reduce the pressure anisotropy by forming magnetic wells (as in Kivelson \& Southwood 1996). ${ }^{5}$ In our test problems with uniform anisotropic plasmas, this is precisely what we find. In the shearing box calculations, however, even at the highest resolutions, we find that the pressure anisotropy becomes so large that equations (33) and (34) are violated and pitch angle scattering due to high-frequency microinstabilities would become important. The resolved mirror modes are thus not able to isotropize the pressure sufficiently fast at all places in the box. ${ }^{6}$ However, it is hard to draw any firm conclusions from these simulations because they stop at around four orbits (for both resolutions $Z l 3$ and $Z h 3$ ) during the initial nonlinear transient stage. At this time the pressure becomes highly anisotropic and becomes very small at some grid points, and the time step limit causes $\delta t \rightarrow 0$.

\section{SUMMARY AND DISCUSSION}

In this paper we have carried out local shearing box simulations of the magnetorotational instability in a collisionless plasma. We are motivated by the application to hot radiatively inefficient flows that are believed to be present in many low-luminosity accreting systems. Our method for simulating the dynamics of a collisionless plasma is fluid-based and relies on evolving a pressure tensor with closure models for the heat flux along magnetic field lines $(\S 2)$. These heat flux models can also be thought of as approximating the collisionless (Landau) damping of linear modes in the simulation.

\footnotetext{
5 At the resolution of $Z l 3$, the fastest growing mirror mode in the computational domain has a linear growth comparable to that of the MRI.

${ }^{6}$ In higher resolution simulations, one can resolve smaller scale and faster growing mirror modes, and thus the effects of isotropization by resolved mirror modes could be come increasingly important. We see no such indications, however, for the range of resolutions we have been able to simulate.
} 
By adiabatic invariance, a slow increase (decrease) in the magnetic field strength tends to give rise to a pressure anisotropy with $p_{\perp}>p_{\|}\left(p_{\|}>p_{\perp}\right)$, where the directions are defined by the local magnetic field. Such a pressure anisotropy can, however, give rise to small-scale kinetic instabilities (firehose, mirror, and ion cyclotron) that act to isotropize the pressure tensor, effectively providing an enhanced rate of pitch angle scattering ("collisions"). We have included the effects of this isotropization via a subgrid model that restricts the allowed magnitude of the pressure anisotropy $(\S 2.3)$.

We find that the nonlinear evolution of the MRI in a collisionless plasma is qualitatively similar to that in MHD, with comparable saturation magnetic field strengths and magnetic stresses. The primary new effect in kinetic theory is the existence of angular momentum transport due to the anisotropic pressure stress (eq. [47]). For the allowed pressure anisotropies estimated in $\S 2.3$, the anisotropic stress is dynamically important and is as large as the Maxwell stress (Table 1). The precise rate of transport in the present simulations is difficult to quantify accurately and depends - at the factor of $\sim 2$ level - on some of the uncertain microphysics in our kinetic analysis (e.g., the rate of heat conduction along magnetic field lines and the exact threshold for pitch angle scattering by small-scale instabilities; see Fig. 7). For better results, it would be interesting to extend these calculations with a more accurate evaluation of the actual nonlocal heat fluxes, equations (11) and (12), or even to directly solve equation (5) for the particle distribution function. Further kinetic studies in the local shearing box, including studies of the small-scale instabilities that limit pressure anisotropy, would be helpful in developing appropriate fluid closures for global simulations.

It is interesting to note that two-temperature RIAFs can only be maintained below a critical luminosity $\sim \alpha^{2} L_{\text {Edd }}$ (Rees et al. 1982). Thus, enhanced transport in kinetic theory due to the anisotropic pressure stress would extend upward in luminosity the range of systems to which RIAFs could be applicable. This is important for understanding, e.g., state transitions in X-ray binaries (e.g., Esin et al. 1997).

In addition to angular momentum transport by anisotropic pressure stresses, Landau damping of long-wavelength modes can be dynamically important in collisionless accretion flows. Because the ZEUS code we employ is nonconservative, we cannot carry out a rigorous calculation of heating by different mechanisms, such as Landau damping and reconnection. Following the total energy-conserving scheme of Turner et al. (2003), however, we estimate that the energy dissipated by Landau damping is comparable to or larger than that due reconnection (which is the major source of heating in MHD simulations). One caveat to this analysis is that in local simulations, the pressure increases in time due to heating, while $B^{2} \sim$ constant. Thus, $\beta$ increases in time and the turbulence becomes more and more incompressible. This will artificially decrease the importance of compressible channels of heating. Clearly it is of significant interest to better understand heating and energy dissipation in RIAFs, particularly for the elec- trons. We will carry out a more a systematic analysis of the energetics of collisionless disks in future global simulations.

In all of our calculations, we have assumed that the dominant source of pitch-angle scattering is high-frequency microinstabilities generated during the growth and nonlinear evolution of the MRI. We cannot, however, rule out that there are other sources of high-frequency waves that pitch angle scatter and effectively decrease the mean free path of particles relative to that calculated here (e.g., shocks and reconnection). As shown in Table 3 and Figure 8, this would decrease the magnitude of the anisotropic stress; we find that for $\nu \gtrsim 30 \Omega$, the results of our kinetic simulations quantitatively approach the MHD limit. In this context it is important to note that the incompressible part of the MHD cascade launched by the MRI is expected to be highly anisotropic with $k_{\perp} \gg k_{\|}$(Goldreich \& Sridhar 1995). As a result, there is very little power in high-frequency waves that could break $\mu$ conservation. It is also interesting to note that satellites have observed that the pressure anisotropy in the solar wind near $1 \mathrm{AU}$ is approximately marginally stable to the firehose instability (Kasper et al. 2002), consistent with our assumption that microinstabilities dominate the isotropization of the plasma.

In this paper we have focused on kinetic modifications to angular momentum transport via anisotropic pressure stresses and parallel heat conduction. In addition, kinetic effects substantially modify the stability of thermally stratified low-collisionality plasmas such as those expected in RIAFs. Balbus (2000) showed that in the presence of anisotropic heat conduction, thermally stratified plasmas are unstable when the temperature decreases outward, rather than when the entropy decreases outward (the usual Schwarzschild criterion). This has been called the magnetothermal instability (MTI). I. J. Parrish \& J. M. Stone (2005) show that in nonrotating atmospheres the MTI leads to magnetic field amplification and efficient heat transport. In future global simulations of RIAFs, it will be interesting to explore the combined dynamics of the MTI, the MRI, and angular momentum transport via anisotropic pressure stresses.

We are grateful to Stephane Ethier for his help with MPI. We thank Shigenobu Hirose for sharing his parallel shearing box version of ZEUS with us. We are grateful to Scott Klasky for his help on visualization. Finally, we thank Ian Parrish for his anisotropic conduction test, and doing comparisons with us. E. Q. is supported in part by NSF grant AST 02-06006, NASA grant NAG5-12043, an Alfred P. Sloan Fellowship, and the David and Lucile Packard Foundation. Part of this research was carried out during a sabbatical by G. W. H. at the University of California at Berkeley, where he is thankful for the hospitality and support of the Miller Institute for Basic Research in Science. This work was also done in part at the Kavli Insitute for Theoretical Physics, supported by the National Science Foundation under grant PHY99-0794.

\section{APPENDIX}

\section{A1. GRID AND VARIABLES}

Figure 9 shows the location of variables on the grid. Scalars and diagonal components of second rank tensors $\left(\rho, p_{\|}\right.$, and $\left.p_{\perp}\right)$ are zone centered. Vectors, representing fluxes out of the box, are located at the cell faces $\left(\boldsymbol{V}, \boldsymbol{B}\right.$, and $\left.\boldsymbol{q}_{\|, \perp}\right)$. The inductive electric field $(\boldsymbol{E})$ is located at cell edges such that the contribution of each edge in calculating $\oint \boldsymbol{E} \cdot \boldsymbol{d l}$ over the whole box cancels, and $\nabla \cdot \boldsymbol{V}=0$ is satisfied to machine precision. The off diagonal part of the pressure tensor in Cartesian coordinates is related to $\Pi=\hat{\boldsymbol{b}} \hat{\boldsymbol{b}}\left(p_{\|}-p_{\perp}\right)$. This is a 


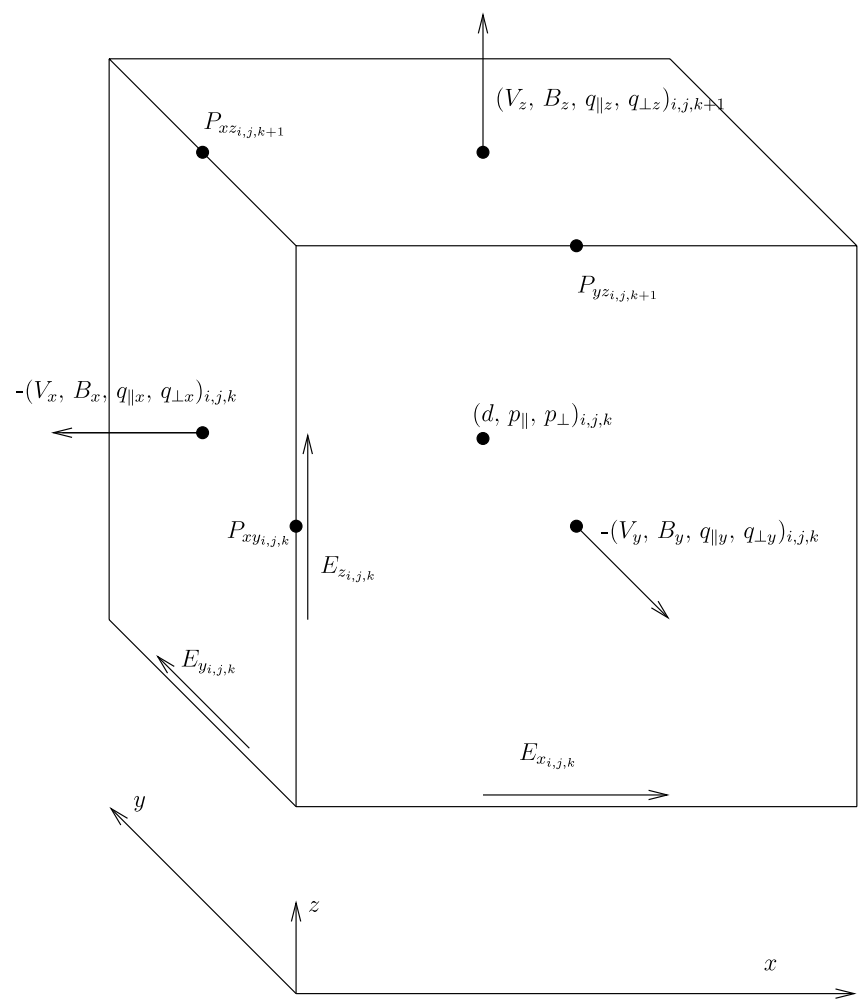

Fig. 9. - Location of different variables on a three-dimensional staggered grid. Vectors $\boldsymbol{V}, \boldsymbol{B}$, and $\boldsymbol{q}_{\|, \perp}$ are located at the face centers. Density $(\rho)$ and diagonal components of the pressure tensor $\left(p_{\perp}, p_{\|}\right)$are located at the zone centers. EMF's $\left(E_{x}, E_{y}, E_{z}\right)$, and off-diagonal components of the pressure tensor $\left(P_{x y}, P_{x z}, P_{y z}\right)$ are located on appropriate edges.

symmetric tensor whose components $P x y, P x z$, and $P y z$ are located such that the finite difference formulae for the evolution of velocities due to off diagonal components of stress are given by

$$
\begin{aligned}
& V x_{i, j, k}{ }^{n+1}=V x_{i, j, k}{ }^{n}-\frac{\delta t}{\delta y}\left(P x y_{i, j+1, k}^{n}-P x y_{i, j, k}^{n}\right)-\frac{\delta t}{\delta z}\left(P x z_{i, j, k+1}^{n}-P x z_{i, j, k}^{n}\right), \\
& V y_{i, j, k}^{n+1}=V y_{i, j, k}{ }^{n}-\frac{\delta t}{\delta x}\left(P x y_{i+1, j, k}^{n}-P x y_{i, j, k}^{n}\right)-\frac{\delta t}{\delta z}\left(P y z_{i, j, k+1}^{n}-P y z_{i, j, k}^{n}\right), \\
& V z_{i, j, k}^{n+1}=V z_{i, j, k}{ }^{n}-\frac{\delta t}{\delta x}\left(P x z_{i+1, j, k}^{n}-P x z_{i, j, k}^{n}\right)-\frac{\delta t}{\delta y}\left(P y z_{i, j+1, k}^{n}-P y z_{i, j, k}^{n}\right),
\end{aligned}
$$

\section{A2. DETERMINATION OF $\delta t$ : STABILITY, AND POSITIVITY}

A time-explicit algorithm must limit the time step in order to satisfy the Courant-Friedrichs-Levy (CFL) stability condition. Physically, $\delta t$ must be smaller than the time it takes any signal (via fluid or wave motion) to cross one grid zone. There is also a limit imposed on $\delta t$ for numerical stability of the diffusive steps. In addition, since there are quantities that must be positive definite $\left(\rho, p_{\|}, p_{\perp}\right)$, we also require $\delta t$ to satisfy positivity. We adopt the following procedure to choose $\delta t$ :

$$
\begin{aligned}
& \delta t_{\mathrm{adv}}=\frac{\min \{\delta x, \delta y, \delta z\}}{\left(|V|+\left|V_{\mathrm{A}}\right|+\left|V_{s}\right|+\left|\Omega L_{x}\right|\right)}, \\
& \delta t_{\|}=\frac{\min \left\{\delta x^{2}, \delta y^{2}, \delta z^{2}\right\}}{2 \kappa_{\|}}, \\
& \delta t_{\perp}=\frac{\min \left\{\delta x^{2}, \delta y^{2}, \delta z^{2}\right\}}{2 \kappa_{\perp}},
\end{aligned}
$$

where $V_{\mathrm{A}}=B /\left[(4 \pi)^{1 / 2}\right]$ is the Alfvén speed, and $V_{s}=\max \left\{\left(3 p_{\|} / \rho\right)^{1 / 2},\left(2 p_{\perp} / \rho\right)^{1 / 2}\right\}$ is the maximum sound speed, taking the anisotropy into account; $\delta t_{\mathrm{adv}}, \delta t_{\|}$, and $\delta t_{\perp}$ correspond to limits on the time step for stability to advection, and parallel and perpendicular heat conduction, respectively.

The source steps for $p_{\|}$and $p_{\perp}$ are given by

$$
\begin{aligned}
& \frac{p_{\|}^{n+1}-p_{\|}^{n}}{\delta t}=\left(-\nabla \cdot \boldsymbol{q}_{\|}-2 p_{\|} \hat{\boldsymbol{b}} \cdot \nabla \boldsymbol{V} \cdot \hat{\boldsymbol{b}}+2 q_{\perp} \nabla \cdot \hat{\boldsymbol{b}}\right)^{n}=A 1, \\
& \frac{p_{\perp}^{n+1}-p_{\perp}^{n}}{\delta t}=\left(-\nabla \cdot \boldsymbol{q}_{\perp \boldsymbol{T}}-p_{\perp} \nabla \cdot \boldsymbol{V}+p_{\perp} \hat{\boldsymbol{b}} \cdot \nabla \boldsymbol{V} \cdot \hat{\boldsymbol{b}}-q_{\perp} \nabla \cdot \hat{\boldsymbol{b}}\right)^{n}=A 2,
\end{aligned}
$$


where $\boldsymbol{q}_{\perp T}=-\kappa_{\perp} \nabla_{\|} T_{\perp}$ denotes the temperature gradient part of $\boldsymbol{q}_{\perp}$. For positivity of $p_{\|}^{n+1}$ and $p_{\perp}^{n+1}$ we require that the following conditions are satisfied: whenever $A 1$ and $A 2$ are negative, $\delta t_{\text {pos }}=\min \left\{-p_{\|}^{n} / A 1,-p_{\perp}^{n} / A 2\right\}$; if $A 1>0, A 2<0$, then $\delta t_{\text {pos }}=-p_{\perp}^{n} / A 2$; if $A 1<0, A 2>0$, then $\delta t_{\text {pos }}=-p_{\|}^{n} / A 1$. Thus, our final constraint on the time step $\delta t$ is given by

$$
\delta t=C_{0} \min \left\{1 /\left[\max \left\{\delta t_{\mathrm{adv}}^{-2}+\delta t_{\|}^{-2}+\delta t_{\perp}^{-2}\right\}\right]^{1 / 2}, \min \left\{\delta t_{\mathrm{pos}}\right\}\right\}
$$

where the max and min are taken over all zones in the box and $C_{0}$ is a safety factor (Courant number), which we take to be 0.5 .

\section{A3. IMPLEMENTATION OF THE PRESSURE ANISOTROPY “HARD WALL”}

If the pressure anisotropy is larger than the constraints given in $\S 2$ by equations (32)-(34), then microinstabilities will turn on that will enhance the pitch-angle scattering rate and quickly reduce the pressure anisotropy to near marginal stability. Because this is a numerically stiff problem, we use an implicit approach, following the treatment of Birn \& Hesse (2001). Whenever equation (32) is violated, we use the following prescription for pitch angle scattering:

$$
\begin{aligned}
& p_{\|}^{n+1}=p_{\|}^{n}-\frac{2}{3} \nu_{p} \delta t\left(\frac{p_{\|}^{n+1}}{2}-p_{\perp}^{n+1}-\frac{B^{2}}{4 \pi}\right), \\
& p_{\perp}^{n+1}=p_{\perp}^{n}+\frac{1}{3} \nu_{p} \delta t\left(\frac{p_{\|}^{n+1}}{2}-p_{\perp}^{n+1}-\frac{B^{2}}{4 \pi}\right),
\end{aligned}
$$

where $\nu_{p}$ is a very large $(\gg 1 / \delta t)$ rate at which marginal stability is approached. This implicit implementation (which can be solved by inverting a $2 \times 2$ matrix) with large $\nu_{p}$ ensures that each time step the pressure anistropy will drop to be very near marginal stability for the firehose instability to break $\mu$ invariance. Given this pitch angle scattering, the collisionality parameter $\nu_{\text {eff }}$ in the thermal conductivity (eqs [42]-[44]) is obtained by comparing equations (A10) and (A11) with equations (38) and (39):

$$
\nu_{\text {eff }}=\max \left\{\nu_{p} \frac{\left[\left(p_{\|}^{n+1} / 2\right)-p_{\perp}^{n+1}-B^{2} / 4 \pi\right]}{\left(p_{\|}^{n+1}-p_{\perp}^{n+1}\right)}, \nu\right\} .
$$

The effective pitch angle scattering rate $\nu_{\text {eff }}$ is independent of $\nu_{p}$ (and much smaller than $\nu_{p}$ ) in the limit of large $\nu_{p}$, and is by definition just large enough to balance other terms in equations (38)-(39) that are trying to increase the pressure anisotropy beyond marginal stability.

The prescriptions for pitch angle scattering due to mirror modes and ion cyclotron waves are similar. For mirror modes we use

$$
\begin{aligned}
& p_{\|}^{n+1}=p_{\|}^{n}-\frac{2}{3} \nu_{p} \delta t\left(p_{\|}^{n+1}-p_{\perp}^{n+1}+2 \xi \frac{p_{\|}^{n+1}}{\beta_{\perp}^{n+1}}\right) \\
& p_{\perp}^{n+1}=p_{\perp}^{n}+\frac{1}{3} \nu_{p} \delta t\left(p_{\|}^{n+1}-p_{\perp}^{n+1}+2 \xi \frac{p_{\|}^{n+1}}{\beta_{\perp}^{n+1}}\right)
\end{aligned}
$$

to limit the pressure anisotropy ( $\xi=3.5$ for our fiducial run $Z l 4)$ and $\nu_{\text {eff }}$ is given by

$$
\nu_{\mathrm{eff}}=\max \left\{\nu_{p} \frac{\left[p_{\|}^{n+1}-p_{\perp}^{n+1}+2 \xi\left(p_{\|}^{n+1} / \beta_{\perp}^{n+1}\right)\right]}{\left(p_{\|}^{n+1}-p_{\perp}^{n+1}\right)}, \nu\right\} .
$$

For ion cyclotron pitch angle scattering we use

$$
\begin{aligned}
& p_{\|}^{n+1}=p_{\|}^{n}-\frac{2}{3} \nu_{p} \delta t\left(p_{\|}^{n+1}-p_{\perp}^{n+1}+S \frac{p_{\|}^{n+1}}{\sqrt{\beta_{\|}^{n+1}}}\right) \\
& p_{\perp}^{n+1}=p_{\perp}^{n}+\frac{1}{3} \nu_{p} \delta t\left(p_{\|}^{n+1}-p_{\perp}^{n+1}+S \frac{p_{\|}^{n+1}}{\sqrt{\beta_{\|}^{n+1}}}\right)
\end{aligned}
$$

and $\nu_{\text {eff }}$ is given by

$$
\nu_{\text {eff }}=\max \left\{\nu_{p} \frac{\left[p_{\|}^{n+1}-p_{\perp}^{n+1}+S\left(p_{\|}^{n+1} / \sqrt{\beta_{\|}^{n+1}}\right)\right]}{\left(p_{\|}^{n+1}-p_{\perp}^{n+1}\right)}, \nu\right\} .
$$




\section{A4. IMPLEMENTATION OF THE ADVECTIVE PART OF $\nabla \cdot \boldsymbol{q}_{\perp}$}

The flux of $p_{\perp}, \boldsymbol{q}_{\perp}=q_{\perp} \hat{b}$, is given by

$$
q_{\perp}=-\kappa_{\perp} \nabla_{\|}\left(\frac{p_{\perp}}{\rho}\right)+\left(\frac{\left(p_{\|}-p_{\perp}\right)}{\rho\left\{\left[(\pi / 2)\left(p_{\|} / \rho\right)\right]^{1 / 2} k_{L}+\nu_{\text {eff }}\right\}} \frac{\boldsymbol{B} \cdot \nabla \boldsymbol{B}}{B^{2}}\right) p_{\perp}=-\kappa_{\perp} \nabla_{\|}\left(\frac{p_{\perp}}{\rho}\right)+V_{\operatorname{mag}} p_{\perp}
$$

where the quantity in square brackets can be thought of as an advection speed due to parallel magnetic gradients. Because of this term, $\boldsymbol{q}_{\perp}$ is not a purely diffusive operator, but also has an advective part characterized by the velocity $V_{\text {mag }}$. If one treats the advective part via a simple central difference method, it does not preserve monotonicity. Instead, to treat the advective part of $\boldsymbol{q}_{\perp}$ properly, we include the advective part in the transport step. After including the advective heat flux in the transport step, it takes the form

$$
\frac{\partial p_{\perp}}{\partial t}+\nabla \cdot\left[\left(\boldsymbol{V}+V_{\mathrm{mag}} \hat{\boldsymbol{b}}\right) p_{\perp}\right]=0 .
$$

Thus, for updating $p_{\perp}$ in the transport step we calculate fluxes on the cell faces using $\boldsymbol{V}+V_{\operatorname{mag}} \hat{\boldsymbol{b}}$ instead of just $\boldsymbol{V}$. The transport step is then directionally split in the three directions. The procedure for monotonicity preserving schemes for calculating fluxes is described in Stone \& Norman (1992a).

\section{A5. ERROR ANALYSIS}

The standard errors in the time averages reported in Table 2 and in Figure 7 are estimated by taking into account the finite correlation time for the physical quantities in the simulation, using techniques recommended by W. Nevins et al. (2006, in preparation). That is, the standard error for the time average $\langle x\rangle=\int d t x(t) / T$ of a signal $x(t)$ is given by $\sigma_{\langle x\rangle}=\left[\operatorname{Var}(x) / N_{\text {eff }}\right]^{1 / 2}$, where $\operatorname{Var}(x)=\int d t(x(t)-\langle x\rangle)^{2} / T$ is the variance of $x, N_{\text {eff }}=T /\left(2 \tau_{\text {int }}\right)$ is the effective number of independent measurements, $T=15$ orbits is the averaging time, and $\tau_{\text {int }}$ is an estimate of the integrated autocorrelation time. There are significant subtleties in determining the integrated autocorrelation time from data. To deal with this, we use a windowing technique as recommended by W. Nevins et al. (2006, in preparation), using $\tau_{\text {int }}=$ $\int_{0}^{T} d \tau C(\tau) W\left(\tau / \tau_{w}\right)$, where $C(\tau)$ is the two-time correlation function from the data, $W\left(\tau / \tau_{w}\right)$ is a smooth window function that cuts off the integral at $\tau \sim \tau_{w}$, and $\tau_{w} \sim\left(T \tau_{\text {int }}\right)^{1 / 2}$ (this gives results insensitive to the choice of window width for $\left.\tau_{\text {int }} \ll T\right)$. Winters et al. (2003) found from comparing three realizations of shearing box MRI simulations that the magnetic stress had a variation of approximately $\pm 6.5 \%$ after averaging over 85 orbits. The simulations we show here were averaged over 15 orbits, so extrapolating from Winters et al. (2003) one might expect the uncertainties to be larger by a factor of $\approx(85 / 15)^{1 / 2} \approx 2.4$. This is consistent with the typical error bars we report in Table 2 and Figure 7.

Balbus, S. A. 2000, ApJ, 534, 420 2004, ApJ, 616, 857

Balbus, S. A., \& Hawley, J. F. 1991, ApJ, 376, 214 1998, Rev. Mod. Phys., 70, 1

Birn, J., \& Hesse, M. 2001, J. Geophys. Res., 106, 3737

Braginskii, S. I. 1965, Rev. Plasma Phys., 1, 205

Chew, C. F., Goldberger, M. L., \& Low, F. E. 1956, Proc. R. Soc. London A, 236, 112

Dimits, A. M., et al. 2000, Phys. Plasmas, 7, 969

Esin, A. A., McClintock, J. E., \& Narayan, R. 1997, ApJ, 489, 865

Gary, S. P., \& Lee, M. A. 1994, J. Geophys. Res., 99, 11297

Gary, S. P., Wang, J., Winske, D., \& Fuselier, S. A. 1997, J. Geophys. Res., 102,27159

Goldreich, P., \& Sridhar, S. 1995, ApJ, 438, 763

Goodman, J., \& Xu, G. 1994, ApJ, 432, 213

Hammett, G. W., Beer, M. A., Dorland, W., Cowley, S. C., \& Smith, S. A. 1993, Plasma Phys. Control. Fusion, 35, 973

Hammett, G. W., Dorland, W., \& Perkins, F. W. 1992, Phys. Fluids B4, 2052 Hammett, G. W., \& Perkins, F. W. 1990, Phys. Rev. Lett., 64, 3019

Hasegawa, A. 1969, Phys. Fluids, 12, 2642

Hawley, J. F., Gammie, C. F., \& Balbus, S. A. 1995, ApJ, 440, 742 (HGB95)

Islam, T., \& Balbus, S. 2005, ApJ, 633, 328

Kasper, J. C., Lazarus, A. J., \& Gary, S. P. 2002, Geophys. Res. Lett, 29, 1839

Kennel, C. F., \& Sagdeev, R. Z. 1967, J. Geophys. Res., 72, 3303

Kivelson, M. G., \& Southwood, D. J. 1996, J. Geophys. Res., 101, 17365

\section{REFERENCES}

Kulsrud, R. M. 1983, Handbook of Plasma Physics, M. N. Rosenbluth, \& R. Z. Sagdeev (New York: North Holland)

McKean, M. E., Gary, S. P., \& Winske, W. D. 1993, J. Geophys. Res., 98, 21313

Narayan, R., Mahadevan, R., \& Quataert, E. 1998, in Theory of Black Hole Accretion Disks, ed. M. A. Abramowicz, G. Bjornsson, \& J. Pringle (Cambridge: Cambridge Univ. Press), 148

Parker, S. E., et al. 1994, Phys. Plasmas, 1, 14611, 1461

Parrish, I. J., \& Stone, J. M. 2005, ApJ, 633, 334

Quataert, E. 2003, in Astron. Nachr., 324, 435

Quataert, E. J., Dorland, W., Hammett, G. W. 2002, ApJ, 577, 524 (QDH02)

Quest, K. B., Shapiro, V. D. 1996, J. Geophys. Res., 101, 24457

Rees, M. J., Phinney, E. S., Begelman, M. C., Blandford, R. D. 1982, Nature, 295, 17

Schekochihin, A. A., Cowley S. C., Kulsrud, R. M., Hammett, G. W., \& Sharma, P. 2005, ApJ, 629, 139

Scott, B. D. 2005, Phys. Plasmas, submitted (physics/0501124)

Sharma, P., Hammett, G. W., \& Quataert E. 2003, ApJ, 596, 1121 (SHQ03)

Snyder, P. B., Hammett, G. W., \& Dorland, W. 1997, Phys. Plasmas, 4, 3974 (SHD97)

Stix, T. H. 1992, Waves in Plasmas (Melville: AIP)

Stone, J. M., \& Norman, M. L. 1992a, ApJS, 80, 753 1992b, ApJS, 80, 819

Turner, N. J., Stone, J. M., Krolik, J. H., \& Sano, T. 2003, ApJ, 593, 992

Winters, W. F., Balbus, S. A., \& Hawley, J. F. 2003, MNRAS, 340, 519 